\title{
Use of an in vitro-in silico testing strategy to predict inter-species and inter-ethnic human differences in liver toxicity of the pyrrolizidine alkaloids lasiocarpine and riddelliine
}

\author{
Jia Ning ${ }^{1} \cdot$ Lu Chen $^{1} \cdot$ Marije Strikwold $^{2} \cdot$ Jochem Louisse $^{1,3} \cdot$ Sebastiaan Wesseling ${ }^{1} \cdot$ Ivonne M. C. M. Rietjens $^{1}$
}

Received: 4 October 2018 / Accepted: 10 January 2019 / Published online: 19 January 2019

(c) The Author(s) 2019

\begin{abstract}
Lasiocarpine and riddelliine are pyrrolizidine alkaloids (PAs) known to cause liver toxicity. The aim of this study was to predict the inter-species and inter-ethnic human differences in acute liver toxicity of lasiocarpine and riddelliine using physiologically based kinetic (PBK) modelling based reverse dosimetry of in vitro toxicity data. The concentration-response curves of in vitro cytotoxicity of lasiocarpine and riddelliine defined in pooled human hepatocytes were translated to in vivo dose-response curves by PBK models developed using kinetic data obtained from incubations with pooled tissue fractions from Chinese and Caucasian individuals, providing PBK models for the average Chinese and average Caucasian, respectively. From the predicted in vivo dose-response curves, the benchmark dose lower and upper confidence limits for $5 \%$ effect $\left(\mathrm{BMDL}_{5}\right.$ and $\left.\mathrm{BMDU}_{5}\right)$ were derived and subsequently compared to those previously obtained in rat to evaluate inter-species differences. The inter-species differences amounted to 2.0-fold for lasiocarpine and 8.2-fold for riddelliine with humans being more sensitive than rats. The inter-ethnic human differences varied 2.0-fold for lasiocarpine and 5.0-fold for riddelliine with the average Caucasian being more sensitive than the average Chinese. In conclusion, the present study provides the proof-of-principle to predict inter-species and inter-ethnic differences in in vivo liver toxicity for PAs by an alternative testing strategy integrating in vitro cytotoxicity data with PBK modelling-based reverse dosimetry.
\end{abstract}

Keywords Physiologically based kinetic $(\mathrm{PBK})$ model $\cdot$ Lasiocarpine $\cdot$ Riddelliine $\cdot$ Inter-species $\cdot$ Inter-ethnic

$\begin{array}{ll}\begin{array}{l}\text { Abbreviations } \\ \mathrm{BMD}_{5}\end{array} & \begin{array}{l}\text { The benchmark dose resulting in 5\% extra } \\ \text { effect }\end{array} \\ \mathrm{BMDL}_{5} & \begin{array}{l}\text { The lower confidence limit of the bench- } \\ \text { mark dose resulting in 5\% extra effect }\end{array} \\ \text { BMDU }_{5} & \begin{array}{l}\text { The upper confidence limit of the bench- } \\ \text { mark dose resulting in 5\% extra effect } \\ \text { Benchmark Response }\end{array}\end{array}$

Electronic supplementary material The online version of this article (https://doi.org/10.1007/s00204-019-02397-7) contains supplementary material, which is available to authorized users.

Jia Ning

jia.ning@wur.nl

1 Division of Toxicology, Wageningen University, Stippeneng 4, 6708 WE Wageningen, The Netherlands

2 Van Hall Larenstein University of Applied Sciences, 8901 BV Leeuwarden, The Netherlands

3 RIKILT Wageningen University and Research, Akkermaalsbos 2, 6708 WB Wageningen, The Netherlands

$\begin{array}{ll}C_{\max } & \text { Maximum concentration } \\ \text { EFSA } & \text { European Food Safety Authority } \\ \text { IARC } & \text { The International Agency for Research on } \\ & \text { Cancer } \\ \text { RED } & \text { Rapid equilibrium dialysis } \\ \text { PAs } & \text { Pyrrolizidine alkaloids } \\ \text { PBK model } & \text { Physiologically based kinetic model } \\ \text { PoDs } & \text { Points of departure } \\ \text { UPLC } & \text { Ultra performance liquid chromatography }\end{array}$

\section{Introduction}

Lasiocarpine and riddelliine (Fig. 1) are chemicals belonging to the group of pyrrolizidine alkaloids (PAs) which are important secondary metabolites of approximately 6000 plant species distributed around the world (Smith and Culvenor 1981). Lasiocarpine is widely distributed in the Heliotropium and Symphytum genus (Boraginaceae species), and riddelliine is present in the genus Senecio (Asteraceae species) (Adams et al. 1942; Smith and Culvenor 1981). 


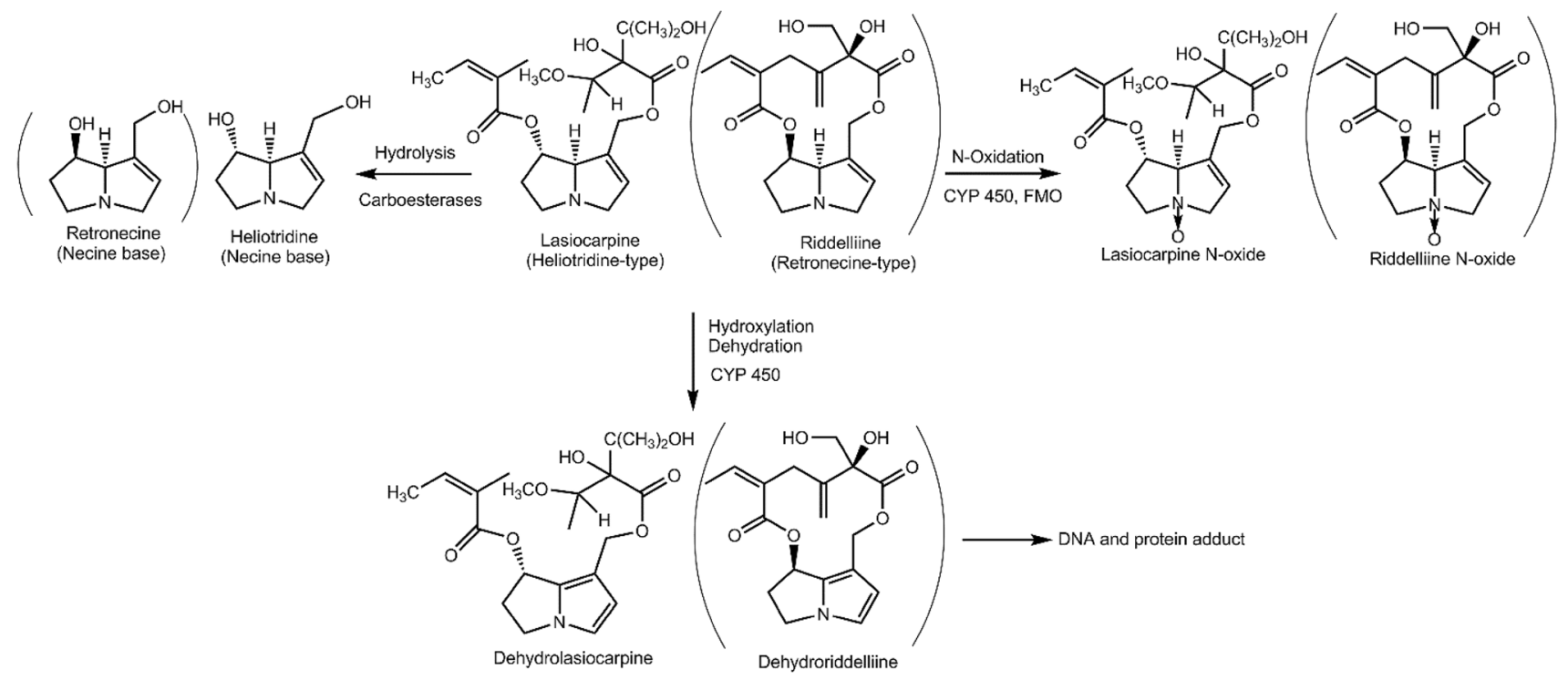

Fig. 1 Schematic presentation of the major metabolic pathways of lasiocarpine (heliotridine-type PA) and riddelliine (retronecine-type PA), the latter shown in brackets. FMO flavin-containing monooxygenase, $C Y P 450$ cytochromes $\mathrm{P} 450$

PA-containing plants can be toxic to livestock, wildlife and humans (Stegelmeier et al. 1999). Human exposure to lasiocarpine and riddelliine may result from the consumption of contaminated food staples, plant food supplements, herbal medicines, herbal teas, honey and milk (EFSA 2011, 2017). Exposure to lasiocarpine, as well as riddelliine has been shown to cause fatal liver veno-occlusive disease in animals and humans (Bane et al. 2012; Mohabbat et al. 1976; Tandon et al. 1978). A 2-year National Toxicology Program (NTP) study testing the genotoxicity and carcinogenicity of lasiocarpine and riddelliine showed that these PAs are also carcinogenic and genotoxic compounds (Chan et al. 2003; NTP 1978, 2003). Liver angiosarcomas were observed in 11 of 23 male rats and 7 of 24 female rats following dietary administration of $0.75 \mathrm{mg} / \mathrm{kg}$ bw/day of lasiocarpine. Riddelliine induced a high incidence of liver hemangiosarcoma in both male ( 43 out of 50 rats) and female rats (38 out of 50 rats) at $0.7 \mathrm{mg} / \mathrm{kg}$ bw/day (Chan et al. 2003; NTP 1978, 2003). The International Agency for Research on Cancer (IARC) classified these alkaloids in Group 2B, potentially carcinogenic to humans (IARC 2017).

Following absorption in the gastrointestinal tract, PAs are distributed to the liver where the main metabolism occurs (Fu et al. 2004). In general, three metabolic pathways have been identified for lasiocarpine (heliotridine-type PA) and riddelliine (retronecine-type PA) (Fig. 1) (EFSA 2011; Fu et al. 2004). The bioactivation pathway is the conversion of lasiocarpine or riddelliine into a toxic intermediate, known as a dehydropyrrolizidine alkaloid, in a reaction mainly catalysed by cytochrome P450 3A4 (Fu et al. 2004). Dehydropyrrolizidine alkaloids react with cellular proteins and DNA leading to liver toxicity, genotoxicity and carcinogenicity (Fu et al. 2004). Other metabolic pathways include hydrolysis and $\mathrm{N}$-oxidation, leading to the formation of necine bases and PA N-oxides, respectively (Fig. 1). These latter two metabolic pathways are considered to lead to detoxification (EFSA 2011).

Significant variations between species regarding the toxicity of PAs have been observed. Recently, Fashe et al. (2015) conducted a comparative study on the metabolism of lasiocarpine by human and rat liver microsomes and found that humans might be more susceptible to lasiocarpineinduced toxicity, because the overall metabolic fingerprint detected upon incubation with human liver microsomes differed from that obtained with rat liver microsomes in the following two major aspects: (1) the amount of detoxified metabolites was lower in incubations with human liver microsomes than with rat liver microsomes, and (2) higher levels of GSH-reactive metabolites were formed in incubations with the human liver microsomes than with the rat liver microsomes. These species differences may be mediated by inter-species differences in biotransformation capacities including CYP 3A4 catalysed bioactivation, carboxylesterase-mediated hydrolysis and the level of cellular GSH (Fashe et al. 2015). In addition to these possible species differences in biotransformation capacities, other studies reported ethnic differences in P450 dependent metabolism and these may potentially result in inter-ethnic differences in PA bioactivation and detoxification. Yang et al. (2012) reported significant differences in phenacetin $O$-deethylation, diclofenac 4'-hydroxylation, $(S)$-mephenytoin 4'-hydroxylation, and chlorzoxazone 6-hydroxylation 
reactions, mediated by CYP 1A2, CYP 2C9, CYP 2C19 and CYP 2E1, respectively between Chinese and Caucasian liver microsomal preparations. Previously, we also reported differences between Chinese and Caucasians regarding the metabolic bioactivation and detoxification of the food-borne genotoxic carcinogen estragole, resulting in a predicted 4.5fold lower formation of the ultimate carcinogenic metabolite of estragole with similar rates of detoxification in Chinese as compared to the Caucasians, and thus a possible lower risk of estragole exposure for the average Chinese at similar levels of exposure (Ning et al. 2017).

Thus, inter-species and inter-ethnic human differences in metabolism might occur and may in theory also affect the sensitivity towards PA toxicity. The aim of this study was to predict the inter-species and inter-ethnic human differences in acute liver toxicity of lasiocarpine and riddelliine using physiologically based kinetic (PBK) modelling-based reverse dosimetry of in vitro toxicity data. Previously, we developed a PBK modelling-based approach to predict acute liver toxicity of lasiocarpine and riddelliine in rats (Chen et al. 2018). The predicted $\mathrm{BMDL}_{5}-\mathrm{BMDU}_{5}$ values for in vivo liver toxicity of lasiocarpine and riddelliine in rats amounted to $23.0-34.4$ and $4.9-8.4 \mathrm{mg} / \mathrm{kg}$ bw/ day, respectively (Chen et al. 2018). That study already showed that the PBK modelling-based approach was able to define inter-compound differences in acute liver toxicity of PAs. The study of Culvenor (1983) estimated that a dose of $0.7-3 \mathrm{mg} / \mathrm{kg}$ bw/day of riddelliine may lead to acute liver toxicity in humans, indicating that humans might be more sensitive than rats towards liver toxicity of PAs. In the present study we developed PBK models for lasiocarpine and riddelliine in different ethnic populations (Chinese and Caucasian) and used these models to convert in vitro toxicity data obtained in pooled human hepatocytes to predict in vivo dose-response data and $\mathrm{BMDL}_{5}-\mathrm{BMDU}_{5}$ values for acute liver toxicity of lasiocarpine and riddelliine in humans. Subsequently, these points of departure (PoDs) were compared to PoDs derived previously for rats by the same methodology (Chen et al. 2018) to evaluate the occurrence of species differences in the metabolism and toxicity of lasiocarpine and riddelliine while the PoDs obtained for the average Chinese and average Caucasian provided insight in inter-ethnic differences.

\section{Materials and methods}

\section{Chemicals and biological materials}

Lasiocarpine (purity $>97 \%$ ) was purchased from Phytolab (Vestenbergsgreuth, Germany). Riddelliine (purity >90\%) was a gift from RIKILT (Wageningen, The Netherlands) and from the National Institute for Environmental Health
Sciences (NIEHS) (Durham, USA). The HepaRG cell line was obtained from Biopredic International (Saint-Grégoire, France). Cryopreserved pooled human hepatocytes (5 mixed gender donors), cryopreserved hepatocyte plating medium (CHPM) (CM 9000) and cryopreserved hepatocyte recovery medium (CHRM) (CM 7000) were purchased from Invitrogen (Cheshire, UK). Willliams medium E (without glutamine), penicillin/streptomycin-solution, L-glutamine, phosphate buffered saline (PBS), and trypsin-EDTA were purchased from Gibco (Paisley, Scotland, UK). Fetal bovine serum was purchased from the American Type Culture Collection (ATCC) (Manassas, USA). Dimethylsulfoxide (DMSO), human insulin and hydrocortisone-21-hemisuccinate were purchased from Sigma-Aldrich (Steinheim, Germany). Potassium dihydrogen phosphate, dipotassium hydrogen phosphate trihydrate, sodium phosphate, sodium chloride and trifluoroacetic acid (TFA) were purchased from VWR International (Darmstadt, Germany). Reduced nicotinamide adenine dinucleotide phosphate (NADPH) was obtained from Roche Diagnostics (Mannheim, Germany). Acetonitrile (UPLC/MS grade) was obtained from Biosolve BV (Valkenswaard, Netherlands). Human plasma and rapid equilibrium dialysis (RED) materials, including RED inserts, RED base plate and sealing tape were obtained from Thermo Fisher Scientific (Bleiswijk, the Netherlands). Pooled Caucasian liver microsomes (33 donors) were purchased from Corning (Woburn, MA, USA). Pooled Caucasian intestinal microsomes (15 donors) were obtained from Xenotech (Lenexa, USA). Pooled Caucasian human lung and kidney microsomes were purchased from Biopredic International (Rennes, France). Pooled Chinese liver microsomes (40 donors) were purchased from PrimeTox (Wuhan, China).

\section{Outline of the PBK modelling-based reverse dosimetry approach in human}

The PBK modelling-based reverse dosimetry approach to predict the in vivo dose-response curves for risk assessment from in vitro cytotoxicity concentration-response data included the following steps: (1) establishment of in vitro cytotoxicity concentration-response curves for lasiocarpine and riddelliine in HepaRG cells and in cryopreserved pooled human hepatocytes from Caucasian donors and correcting for toxicity expected in hepatocytes from the Chinese population as described below in the "UPLC and LC-MS/MS analysis" section, (2) development of PBK models for lasiocarpine and riddelliine in human using the kinetic parameters defined in the present study using both Chinese and Caucasian human liver subcellular factions, (3) translation of in vitro concentration-response curves to the in vivo dose-response curves using the PBK models, (4) $\mathrm{BMD}$ analysis based on the predicted in vivo dose-response curves to obtain the $\mathrm{BMDL}_{5}$ and $\mathrm{BMDU}_{5}$ values as points 
of departure (PoDs), (5) comparison of the predicted PoDs in human (Chinese and Caucasian) to one another and to the previously predicted PoDs in rats (Chen et al. 2018) to assess for inter-ethnic and inter-species differences in acute liver toxicity.

\section{In vitro liver toxicity data}

Two types of cells were used to determine the in vitro concentration-response curves for acute cytotoxicity in human liver including cells from the HepaRG cell line and human hepatocytes. The HepaRG cell line is a human hepatoma cell line, which can differentiate into hepatocyte-like morphology by treatment with DMSO (Kanebratt and Andersson 2008). HepaRG cells were seeded at a density of 9000 cells/well in 96-well plates in growth medium consisting of Williams' medium E, supplemented with $10 \%$ fetal bovine serum (FCS), $100 \mathrm{IU} / \mathrm{ml}$ penicillin, $100 \mu \mathrm{g} / \mathrm{ml}$ streptomycin, $1 \%(\mathrm{v} / \mathrm{v}) \mathrm{L}$-glutamine, $5 \mu \mathrm{g} / \mathrm{ml}$ human insulin and $50 \mu \mathrm{M}$ hydrocortisone-21-hemisuccinate. The medium was renewed every 2-3 days. After 2 weeks, the cells were nearly confluent and were cultured in the differentiation medium supplemented with $1.7 \%(\mathrm{v} / \mathrm{v})$ DMSO. The medium was refreshed every 2-3 days. After 2 weeks differentiation treatment, HepaRG cells were fully differentiated and were used for experiments within 4 weeks with refreshing the medium every $2-3$ days according to the protocol provided by Biopredic International.

Cryopreserved mixed gender primary human hepatocytes from five Caucasian donors were also used to perform the cytotoxicity experiments and define in vitro concentration-response curves. Cryopreserved human hepatocytes were thawed according to the protocol provided by Invitrogen. Briefly, human hepatocytes were thawed in a $37{ }^{\circ} \mathrm{C}$ water bath and transferred to $50 \mathrm{ml}$ thawing medium $(\mathrm{CM}$ 7000). The cells were centrifuged at room temperature at $100 \mathrm{~g}$ for $10 \mathrm{~min}$ and re-suspended in the plating medium (CM 9000). After determination of the cell viability using trypan blue exclusion, the cells were seeded in 96-well plates at a density of 50,000 cells/well and incubated in a $5 \% \mathrm{CO}_{2}$ : 95\% air-humidified incubator for $6 \mathrm{~h}$, after which the cells were ready to be exposed to the compounds. Only batches with a cell viability $>90 \%$ were used for experiments.

Cytotoxicity was determined with the MTT assay by assessing the activity of mitochondrial dehydrogenase enzymes according to the method of Field et al. (2015). In brief, the fully differentiated HepaRG cells and human hepatocytes were exposed to serum-free medium containing different concentrations of lasiocarpine and riddelliine ranging from 4 to $300 \mu \mathrm{M}$ (final concentrations), added from 200 times concentrated stock solutions in DMSO. Each concentration was measured in triplicate and three independent experiments were performed. A solvent control was included in each independent experiment. After $24 \mathrm{~h}$ exposure, MTT $(0.45 \mathrm{mg} / \mathrm{ml}$ final concentration) was added and cells were incubated for another $2 \mathrm{~h}$ in the incubator. The formed MTT formazan crystals were dissolved in DMSO and the absorbance was measured at a microplate reader at $562 \mathrm{~nm}$ (SpectraMax M2, USA). All the data are presented as mean values $\pm \mathrm{SD}$. The half maximal inhibitory concentration $\left(\mathrm{IC}_{50}\right)$ values used to assess the in vitro acute liver toxicity of lasiocarpine and riddelliine were obtained using GraphPad Prism 5.04 (GraphPad software, San Diego California U.S.A.) using a non-linear regression model for curve fitting $\mathrm{Y}=$ Bottom $+($ Top-Bottom $) /\left(1+10^{\wedge}(\mathrm{X}-\operatorname{LogIC} 50)\right)$. The in vitro concentration-response curve for liver toxicity for Chinese hepatocytes was obtained as described in the "UPLC and LC-MS/MS analysis" section.

\section{In vitro microsomal incubations}

The in vitro incubations for the conversion of lasiocarpine with Chinese and Caucasian liver microsomes were performed as described by Fashe et al. (2015). Briefly, mixed gender human liver microsomes were incubated with lasiocarpine in the presence of NADPH. The incubation mixtures contained (final concentrations) $2 \mathrm{mM}$ NADPH and $0.04 \mathrm{mg} / \mathrm{ml}$ microsomal protein in $0.1 \mathrm{M}$ potassium phosphate ( $\mathrm{pH}$ 7.4). After pre-incubation for $5 \mathrm{~min}$, the reaction was started by adding NADPH. Incubations were performed for $30 \mathrm{~min}$ at substrate concentrations ranging from 3 to $200 \mu \mathrm{M}$ (final concentrations) (added from 100 times concentrated stock solutions in DMSO), after which the reaction was terminated by adding ice-cold acetonitrile $(20 \% \mathrm{v} / \mathrm{v})$. Under these conditions conversion of lasiocarpine was linear in time (1-30 min) and with the amount of microsomal protein $(0.01-0.04 \mathrm{mg} / \mathrm{ml}$ ) (data not shown). Blank incubations were performed in the absence of the cofactor NADPH.

The incubations for metabolism of riddelliine by human liver microsomes contained (final concentrations) $2 \mathrm{mM}$ $\mathrm{NADPH}, 0.5 \mathrm{mg} / \mathrm{ml}$ microsomal protein for Chinese and Caucasian samples in $0.1 \mathrm{M}$ potassium phosphate ( $\mathrm{pH} 7.4)$ and 3 to $400 \mu \mathrm{M}$ (final concentrations) riddelliine added from 100 times concentrated stock solutions in DMSO. After 5 min pre-incubation at $37^{\circ} \mathrm{C}$, the reactions were started by adding NADPH. Incubations were carried out for $30 \mathrm{~min}$ and the reaction was terminated by adding ice-cold acetonitrile $(20 \% \mathrm{v} / \mathrm{v})$. Under these conditions conversion of riddelliine was linear in time (1-30 min) and with the amount of microsomal protein $(0.04-0.5 \mathrm{mg} / \mathrm{ml})$ (data not shown). Blank incubations were performed in the absence of the cofactor NADPH.

To compare the inter-ethnic differences in metabolic activation of lasiocarpine and riddelliine, incubations with Chinese and Caucasian liver microsomes were performed as described above in the presence of $20 \mathrm{mM}$ glutathione 
(GSH) to scavenge dehydropyrrolizidine alkaloid metabolites. The level of GSH in the incubations was optimized to obtain the maximum scavenging of dehydropyrrolizidine alkaloid metabolites as major glutathione conjugate, namely 7-GS-DHP (Tamta et al. 2012), which was analyzed by LC-MS/MS analysis as described in the "UPLC and LC-MS/MS analysis" section.

The kinetic constants for conversion of lasiocarpine and riddelliine by Caucasian intestinal, lung and kidney microsomal preparations were determined as described above for liver microsomes but increasing the incubation time to $2 \mathrm{~h}$. Under these conditions conversion of lasiocarpine and riddelliine was linear with time (1-120 min) and with the amount of microsomal protein added $(0.01-0.04 \mathrm{mg} /$ $\mathrm{ml}$ for lasiocarpine, $0.04-0.5 \mathrm{mg} / \mathrm{ml}$ for riddelliine) for the intestine, whereas negligible conversion was observed for lung and kidney (data not shown). Before Ultra Performance Liquid Chromatography photodiode array analysis (UPLC-PDA) or LC-MS/MS analysis, all samples were centrifuged for $5 \mathrm{~min}$ at $15,000 \mathrm{~g}$ to precipitate microsomal proteins. Intestine, lung and kidney microsomes from the Chinese population are not available. However, the kinetic parameters obtained using pooled intestinal microsomal preparations from Caucasian individuals were used for the Chinese PBK model, because the results of the sensitivity analysis (see "Sensitivity analysis" section) revealed that the parameters related to the intestinal metabolism of lasiocarpine and riddelliine do not have a large influence on the predicted maximum concentration in the liver blood $\left(C_{\max }\right)$. Because conversion by kidney and lung microsomes was negligible (see result section), no further kinetic data for these organs were required.

The substrate depletion approach was used to obtain the kinetic parameters. The amount of substrate depletion of lasiocarpine and riddelliine was determined as the amount detected in the blank incubations in the absence of the cofactor NADPH minus the amount detected in the incubations with NADPH. The amounts of 7-GS-DHP formed were corrected for the amounts detected in the blank incubations performed without cofactor NADPH. The data for the rate of conversion of lasiocarpine and riddelliine with increasing substrate concentration [S] were fitted to the standard Michaelis-Menten Eq. (1):

$v=V_{\max } /\left(1+K_{m} /[S]\right)$

The apparent maximum velocity $\left(V_{\max }\right)$ and the apparent Michaelis-Menten constant $\left(K_{\mathrm{m}}\right)$ were determined by fitting the data to this equation using GraphPad Prism version 5.04 (GraphPad software, San Diego California, USA). $V_{\max }$ values expressed as $\mathrm{nmol} / \mathrm{min} /(\mathrm{mg}$ microsomal protein) were scaled to the $V_{\max }$ expressed as $\mu \mathrm{mol} / \mathrm{h} / \mathrm{kg}$ bw using microsomal protein yields of $35 \mathrm{mg} / \mathrm{g}$ liver (Medinsky et al. 1994) and $20.6 \mathrm{mg} / \mathrm{g}$ small intestine (Cubitt et al.
2009), bodyweight and tissue fractions of human (Chinese and Caucasian). The $K_{\mathrm{m}}$ values determined in vitro were assumed to be equal to in vivo $K_{\mathrm{m}}$ values.

\section{Protein binding correction}

To correct for protein binding in the in vivo situation in human liver blood, while the in vitro toxicity was determined in serum-free medium, the fraction of unbound lasiocarpine and riddelliine under the in vivo conditions $\left(f_{\mathrm{ub}}\right)$ was determined. To this end, the $f_{\mathrm{ub}}$ for both compounds in human blood was determined using the RED (rapid equilibrium dialysis) device. According to the protocol described by Waters et al. (2008), $300 \mu \mathrm{l}$ of spiked human plasma containing $5 \mu \mathrm{M}$ lasiocarpine and 5 or $50 \mu \mathrm{M}$ riddelliine (final concentration, $1 \% \mathrm{v} / \mathrm{v}$ DMSO) were added to the plasma chambers of the RED device insert, while $500 \mu$ dialysis buffer (PBS containing $100 \mathrm{mM}$ sodium phosphate and $150 \mathrm{mM}$ sodium chloride) were added to the buffer chamber. The device was sealed with tape and incubated at $37{ }^{\circ} \mathrm{C}$ on a shaker at $250 \mathrm{rpm}$ for $5 \mathrm{~h}$. After the system reached equilibrium, $50 \mu \mathrm{l}$ of post-dialysis samples were collected from the plasma and buffer chambers into separate tubes. Subsequently, $50 \mu \mathrm{l}$ of human plasma was added to the buffer samples and the same volume of dialysis buffer was added to the plasma samples, after which protein was precipitated by addition of ice-cold acetonitrile $(75 \% \mathrm{v} / \mathrm{v})$. After incubation on ice for $30 \mathrm{~min}$, the mixtures were centrifuged at $15,000 \mathrm{~g}$ for $15 \mathrm{~min}$ at $4{ }^{\circ} \mathrm{C}$, and the supernatants were analyzed by UPLC-PDA. The measurements were performed in three independent experiments.

The fraction of unbound lasiocarpine and riddelliine in human blood $\left(f_{\mathrm{ub}}\right)$ was assumed to be the same as in human plasma in the present study and was defined as the ratio of the concentration of the test compound in the buffer chamber and the concentration in the plasma chamber (van Liempd et al. 2011; Waters et al. 2008). The $f_{\text {ub }}$ thus obtained was used to correct for the difference in protein binding in the in vitro assay and the in vivo situation when performing the PBK modelling-based reverse dosimetry. To this end, the effect concentration of lasiocarpine and riddelliine in human liver blood $\left(C_{\mathrm{Lc} / \mathrm{Rd} \text {, human blood }}\right)$ used for reverse dosimetry was corrected by the following Eq. (2):

$C_{L c / R d, \text { human blood }}=C_{u b, \text { in vitro }} / f_{u b}$,

where $f_{\mathrm{ub}}$ is the fraction of the unbound lasiocarpine and riddelliine in human blood and $C_{\mathrm{ub}}$ in vitro is the unbound concentration of lasiocarpine and riddelliine in the in vitro culture medium. Since the exposure medium for the HepaRG and human hepatocyte cell lines was serum free, the concentrations of lasiocarpine and riddelliine in the in vitro cytotoxicity assay were considered to be the unbound 
concentration. This correction implies that the unbound fraction in vitro is set equal to the unbound fraction in vivo.

\section{UPLC and LC-MS/MS analysis}

The supernatant of each sample was analyzed on UPLC using a BEH C18 $(1.7 \mu \mathrm{m} 2.1 \times 50 \mathrm{~mm})$ column with a guard column and a photodiode array (PDA) detector (Acquity, Waters). The flow rate was $0.6 \mathrm{ml} / \mathrm{min}$ and the mobile phase consisted of ultrapure water with $0.1 \%(\mathrm{v} / \mathrm{v})$ TFA and acetonitrile. For the analysis of lasiocarpine, a gradient was applied from 0 to $50 \%$ acetonitrile in ultrapure water in $2 \mathrm{~min}$, after which the percentage of acetonitrile was increased to $100 \%$ in 3 min and kept at this level for 2 min longer, after which the column was set back to the starting conditions. Under these conditions, lasiocarpine had a retention time of $1.9 \mathrm{~min}$. For detection of riddelliine, a linear gradient from 0 to $50 \%$ acetonitrile in ultrapure water during 4 min was applied, followed by an increase to $100 \%$ acetonitrile in $1 \mathrm{~min}$. This percentage was kept for $1 \mathrm{~min}$ to wash the column, after which the column was set back to the starting conditions. Under these conditions, riddelliine eluted at $1.8 \mathrm{~min}$. Identification of lasiocarpine and riddelliine was achieved by comparison of the UV spectra and retention times of these compounds to those of reference compounds. Quantification of lasiocarpine and riddelliine was done by comparing the peak areas of these compounds at a wavelength of $220 \mathrm{~nm}$ to the calibration curve of the corresponding standard compounds.

The major glutathione conjugate of lasiocarpine and riddelliine, 7-GS-DHP was analyzed and quantified by LC-MS/ MS. LC-MS/MS analysis was performed on a Shimadzu Nexera XR LC-20AD SR UPLC system coupled with a Shimadzu LCMS-8040 mass spectrometer (Kyoto, Japan). The samples $(1 \mu \mathrm{l})$ were loaded onto a reverse phase C18 column $(1.7 \mu \mathrm{m} 2.1 \times 50 \mathrm{~mm})$ with a flow rate of $0.3 \mathrm{ml} /$ min. The column temperature was set to $40{ }^{\circ} \mathrm{C}$. The mobile phase consisted of ultrapure water with $0.1 \%$ (v/v) formic acid and acetonitrile containing $0.1 \%(\mathrm{v} / \mathrm{v})$ formic acid. The gradient began with $0 \%$ acetonitrile for one minute to wash away unwanted salts, followed by a linear gradient from 0 to $5 \%$ in $8 \mathrm{~min}$ and a further increase to $100 \%$ in $6 \mathrm{~min}$. This percentage was kept for $0.5 \mathrm{~min}$, after which the column was set back to the starting conditions. A Shimadzu LCMS-8040 triple quadrupole with electrospray ionization (ESI) interface was used to perform the MS-MS analysis. The instrument was operated in positive mode in the multiple reaction monitoring (MRM) mode with a spray voltage of $4.5 \mathrm{KV}$. The 7-GS-DHP was monitored at the $[\mathrm{M}+\mathrm{H}]^{+}$of precursor to product $443.2 \rightarrow 425.15(\mathrm{CE}=-7 \mathrm{kV}), 443.2 \rightarrow 118.1$ $(\mathrm{CE}=-24 \mathrm{kV})$ and $443.2 \rightarrow 247.2(\mathrm{CE}=-15 \mathrm{kV}) \mathrm{m} / \mathrm{z}$. Some of these MRMs were also used in the study of Tamta et al. (2012). Under these conditions, 7-GS-DHP eluted at
$8.97 \mathrm{~min}$ and the chromatogram of 7-GS-DHP is presented in the supplementary materials 1 . The peak area of the total ion chromatogram (TIC) of 7-GS-DHP was acquired using Postrun analysis in the software LabSolution (Shimadzu). The ratio of the peak areas of 7-GS-DHP formed in incubations with Chinese and Caucasian liver microsomes at each substrate concentration, was assumed to reflect the interethnic differences in metabolic activation of lasiocarpine and riddelliine. Thus, these ratios were used to convert the concentration-response curve for in vitro toxicity obtained with human hepatocytes derived from Caucasian donors to a curve for Chinese donors. To this end, the percentage of cytotoxicity at each concentration was multiplied by the amount of 7-GS-DHP formed in incubations with Caucasian liver samples divided by the amount formed in similar incubations with Chinese liver microsomes. This provided an estimated concentration-response curve for cytotoxicity towards Chinese liver hepatocytes.

\section{Development of a PBK model for lasiocarpine and riddelliine in human}

PBK models for lasiocarpine and riddelliine for the average Chinese and average Caucasian were developed based on the PBK models previously defined and evaluated by Chen et al. (2018) for lasiocarpine and riddelliine in rats. The structure of the model for lasiocarpine and riddelliine is presented in Fig. 2. The model has 7 compartments including blood, fat, rapidly perfused tissue, slowly perfused tissue, liver and intestine that are mutually connected through the systemic circulation which is

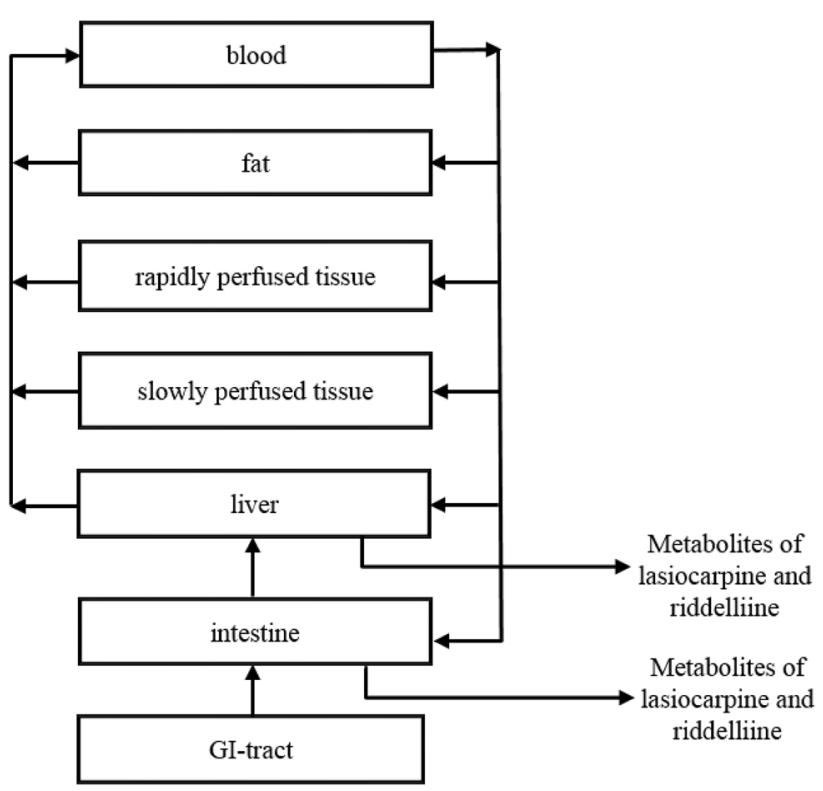

Fig. 2 Schematic diagram of the PBK models for lasiocarpine and riddelliine in human 
represented by a blood compartment. The intestine and liver compartments are included in the model, since the conversion of lasiocarpine and riddelliine occurs in these organs. The physiological parameters for the average Chinese and average Caucasian were derived from the literature (Brown et al. 1997; NHFPC 2007, 2014) and are presented in Table S2 in the supplementary materials 2. Several reports from the literature indicate that Chinese have significantly smaller livers than Caucasians (Chan et al. 2006; Shi et al. 2009; Yuan et al. 2008). The physiological parameters provided by the National Health Commission of the People's Republic of China (NHFPC) also show that the Chinese liver volume is 1.3 -fold lower than the Caucasian liver volume. The tissue:blood partition coefficients were estimated from $\log K_{\text {ow }}$ according to the method described by DeJongh et al. (1997). The log $K_{\text {ow }}$ values for lasiocarpine and riddelliine were estimated by ChemBio 3D 2010 software (CambrigeSoft, USA).

Lasiocarpine and riddelliine were assumed to enter the body from the gastrointestinal tract lumen following first-order uptake. The in vivo oral absorption rate constant $(\mathrm{Ka})$ of lasiocarpine and riddelliine in human were assumed to be the same as previously determined (Chen et al. 2018). The Ka obtained for lasiocarpine was $1.55 / \mathrm{h}$ and for riddelliine the value amounted to $0.72 / \mathrm{h}$. Model equations were coded (supplementary materials 3) and numerically integrated in Berkeley Madonna 8.0.1 (Macey and Oster, UC Berkeley, CA, USA) using the Rosenbrock's algorithm for stiff systems.

\section{Sensitivity analysis}

A sensitivity analysis was performed to identify which parameters have the largest influence on the model predictions. Normalized sensitivity coefficients (SC) were determined using the following Eq. (3) for the prediction of maximum liver blood concentration $\left(C_{\max }\right)$ of lasiocarpine and riddelliine:

$S C=\left(C^{\prime}-C\right) /\left(P^{\prime}-P\right) \times(P / C)$,

where $C$ is the initial value of the model output $\left(C_{\max }\right.$ in liver blood); $C^{\prime}$ is the modified model output resulting from a 5\% increase of the parameter value; $P$ is the initial parameter value; $P$ ' is the modified parameter value (Evans and Andersen 2000). A 5\% increase in parameter values was chosen to analyze the effect of a change in parameter values on $C_{\max }$ of lasiocarpine and riddelliine at a dose of $8 \mathrm{ng} / \mathrm{kg}$ bw/day and $3 \mathrm{mg} / \mathrm{kg}$ bw/day for $24 \mathrm{~h}$ exposure, representing respectively an average daily intake of PAs via consumption of herbal tea (Bfr 2013) and a dose level known to cause liver toxicity in humans (Culvenor 1983). Each parameter was analyzed individually while other parameters were kept at their initial value.

\section{Translation of the in vitro concentration-response curves to in vivo dose-response curves}

The in vitro concentration-response curves on acute cytotoxicity were translated to in vivo dose-response curves on hepatotoxicity of lasiocarpine and riddelliine by PBK modelling-based reverse dosimetry. For this translation, each concentration applied in the cytotoxicity experiments was converted to a corresponding $C_{\mathrm{Lc} / \mathrm{Rd} \text {, human blood }}$ which was then assumed to be the $C_{\max }$ (the maximum concentration) of lasiocarpine and riddelliine in the liver blood and used as input in the PBK model to determine the corresponding oral dose which would result in acute hepatotoxicity. By performing this exercise for each concentration used in the in vitro cytotoxicity assay, the concentration-response curve was translated to an in vivo dose-response curve.

The in vitro concentration-response curve on cytotoxicity of lasiocarpine and riddelliine for the average Chinese was obtained based on in vitro concentration-response curves on cytotoxicity derived from mixed gender human (Caucasian) liver hepatocytes, correcting the curve for the differences in bioactivation between Chinese and Caucasians as explained above the "UPLC and LC-MS/MS analysis" section. This was done because mixed gender Chinese liver hepatocytes were not available. To this end, each concentration in the cytotoxicity experiment was first multiplied by the fold difference in the peak area of the major glutathione conjugate, 7-GS-DHP formed in liver microsomal incubations in the presence of GSH for Chinese and Caucasian samples. Subsequently, the corrected in vitro concentration-response curves for Chinese were translated to the in vivo dose-response curves based on the same approach as described above for Caucasians using the PBK model for the average Chinese.

\section{BMD analysis of predicted in vivo dose-response data}

BMD modelling was applied on the predicted in vivo dose-response curves to derive the $\mathrm{BMDL}_{5}-\mathrm{BMDU}_{5}$ (lower-upper 90\% confidence interval of the benchmark dose that gives a 5\% response) for hepatotoxicity by the PROAST software version 38.9 developed by RIVM (the Dutch National Institute for Public Health and the Environment) (Slob 2002). This BMD modelling was performed using Exponential and Hill models for continuous data. Models were selected if they passed the goodness-of-fit test at $P=0.05$ and the ratio of the $\mathrm{BMD}_{5}$ and $\mathrm{BMDL}_{5}$ was not more than threefold (EPA 2017). 


\section{Results}

\section{In vitro cytotoxicity}

Figure 3 shows the concentration-response curves for the cytotoxicity of lasiocarpine and riddelliine in HepaRG cells and human pooled hepatocytes from Caucasian donors. Table 1 shows the $\mathrm{IC}_{50}$ values derived from these concentration-response curves. The $\mathrm{IC}_{50}$ for the cytotoxicity of lasiocarpine and riddelliine towards rat hepatocytes reported in the previous study of Chen et al. (2018) are also shown in Table 1 for comparison. The results show that rat hepatocytes are more sensitive to lasiocarpine and riddelliine than the HepaRG and human hepatocytes, with the $\mathrm{IC}_{50}$ of lasiocarpine in rat hepatocytes being 20- and 2-fold lower than the $\mathrm{IC}_{50}$ in HepaRG and human hepatocytes, respectively and the $\mathrm{IC}_{50}$ of riddelliine in rat hepatocytes being 22- and 7-fold lower than the $\mathrm{IC}_{50}$ for riddelliine in HepaRG and human hepatocytes, respectively. Therefore, the sensitivity towards the toxicity of lasiocarpine and riddelliine decreases in the order: rat hepatocytes $>$ human hepatocytes $>$ HepaRG cells. In the different cell models the cytotoxicity of lasiocarpine and riddelliine is comparable. For HepaRG cells the $\mathrm{IC}_{50}$ of lasiocarpine is 1.7-fold higher than that of riddelliine while for human hepatocytes, the $\mathrm{IC}_{50}$ of lasiocarpine is 2.1-fold lower than that of riddelliine. Chen et al. (2018) already reported that the $\mathrm{IC}_{50}$ of lasiocarpine was 1.8-fold higher than that of riddelliine in rat hepatocytes.

Given that Chinese human liver hepatocytes are not available, the cytotoxicity of lasiocarpine and riddelliine towards Chinese liver hepatocytes was modelled based on the concentration-response curves obtained with mixed gender Caucasian liver hepatocytes, making a correction for the relative difference in bioactivation as determined in in vitro incubations. Figure 3 also shows the in vitro concentration-response curves of lasiocarpine and riddelliine for the Chinese population obtained in this way. From Fig. 3, the $\mathrm{IC}_{50}$ of lasiocarpine and riddelliine were derived for the average Chinese amounting to $126 \pm 39 \mu \mathrm{M}$ and $380 \pm 67 \mu \mathrm{M}$, respectively. Comparing these $\mathrm{IC}_{50}$ values to the values obtained for the average Caucasian (Table 1), reveals that the $\mathrm{IC}_{50}$ values of lasiocarpine and riddelliine for the Chinese are estimated to be 6.0-fold and 8.6-fold higher, respectively. The cytotoxicity data are presented in the supplementary materials 4 .

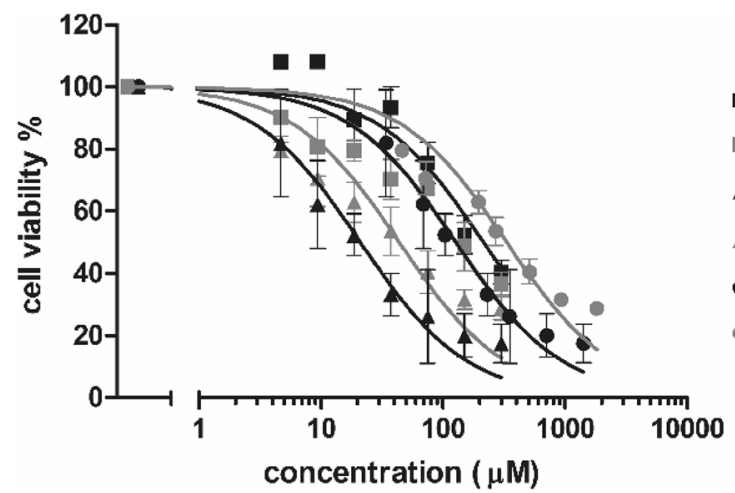

- HepaRG, lasiocarpine

- HepaRG, riddelliine

- Human (Caucasian) hepatocyte, lasiocarpine

- Human (Caucasian) hepatocyte, riddelliine

- Corrected Human (Chinese) hepatocyte, lasiocarpine

- Corrected Human (Chinese) hepatocyte, riddelline

Fig. 3 Concentration-response curves for the cytotoxicity of lasiocarpine (black lines) and riddelliine (grey lines) in HepaRG cells (squares), human (Caucasian) hepatocytes (triangles) and corrected human (Chinese) hepatocytes (circles) upon $24 \mathrm{~h}$ exposure (mean values \pm SD)

Table $1 \mathrm{IC}_{50}$ values for the cytotoxicity of lasiocarpine and riddelliine in different cell models

\begin{tabular}{lcl}
\hline Cell line & $\mathrm{IC}_{50}(\mu \mathrm{M}) \pm \mathrm{SD}$ & Studies \\
\hline Lasiocarpine & & \\
HepaRG cells & $215 \pm 47$ & Present study \\
Human (Caucasian) hepatocytes & $21 \pm 7$ & Present study \\
Corrected human (Chinese) hepatocytes & $126 \pm 39$ & Present study \\
Rat hepatocytes & $11 \pm 2$ & Chen et al. (2018) \\
Riddelliine & & \\
HepaRG cells & $130 \pm 30$ & Present study \\
Human (Caucasian) hepatocytes & $44 \pm 9$ & Present study \\
Corrected human (Chinese) hepatocytes & $380 \pm 67$ & Present study \\
Rat hepatocytes & $6 \pm 1$ & Chen et al. (2018) \\
\hline
\end{tabular}




\section{Microsomal conversion of lasiocarpine and riddelliine by Chinese and Caucasian tissue fractions}

Upon incubation of increasing concentrations of lasiocarpine and riddelliine with Chinese and Caucasian tissue fractions and NADPH, the extent of substrate depletion was quantified by UPLC-PDA analysis. Table 2 displays the apparent $V_{\max }$ and $K_{\mathrm{m}}$ values obtained from the plots as shown in Fig. 4 and the catalytic efficiency, calculated as $V_{\max } / K_{\mathrm{m}}$. Conversion of lasiocarpine and riddelliine was detected in incubations with liver and intestinal microsomes. Incubations of lasiocarpine and riddelliine with lung and kidney microsomes of Caucasians did not result in detectable metabolic conversion. Lung and kidney microsomes from Chinese individuals were not available, but it was assumed that they would also not cause metabolic conversion of lasiocarpine and riddelliine.

Liver was the major organ for lasiocarpine and riddelliine metabolism, as reflected by the fact that the catalytic efficiency for the depletion of lasiocarpine or riddelliine in incubations with pooled liver microsomes from Caucasian individuals is around 15-fold higher than the catalytic efficiency derived from incubations with intestinal microsomes. Comparison of the kinetic constants for lasiocarpine and riddelliine reveals that for depletion of lasiocarpine by Chinese liver fractions, the $V_{\max }$ is 15.3 -fold higher and the $K_{\mathrm{m}}$ is 3.7-fold lower than for riddelliine, resulting in a 54.8-fold higher catalytic efficiency for lasiocarpine metabolism. For Caucasian liver samples, the $V_{\max }$ and $K_{\mathrm{m}}$ for depletion of lasiocarpine are 2.6-fold higher and 10.5-fold lower, respectively, than those of riddelliine, resulting in a 27.1 -fold higher catalytic efficiency for depletion of lasiocarpine. In the case of the intestine, the apparent $V_{\max }$ and $K_{\mathrm{m}}$ for the depletion of lasiocarpine in incubations with small intestinal microsomes from Caucasians are 5.0-fold higher and 5.3-fold lower, respectively, than those of riddelliine. The catalytic efficiency for depletion of lasiocarpine in these intestinal incubations is 25 -fold higher than for depletion of riddelliine.

Comparison of the kinetic data for the depletion of lasiocarpine and riddelliine by liver fractions from the Chinese and Caucasians reveals that the catalytic efficiency for both compounds by the Chinese liver samples is $2.3-4.6$-fold lower, which is mainly due to a 2.0 -fold higher $K_{\mathrm{m}}$ for depletion of lasiocarpine and 6.7-fold lower $V_{\max }$ for depletion of riddelliine in Chinese liver. Comparison of the kinetic data for formation of 7-GS-DHP of both compounds by Chinese and Caucasian liver fractions, reveals that over the substrate concentration range used in incubations with Chinese liver microsomes bioactivation of lasiocarpine and riddelliine is 7.5-fold and 8.1-fold lower, respectively. $V_{\max }$ values in these incubations were not quantified because for correction of the in vitro curves only the relative ratio in bioactivation were required. The curves of the concentration-dependent rate of formation of 7-GS-DHP of both compounds, expressed in peak area/min $/ \mathrm{mg}$ liver protein and the ratio of the peak areas of 7-GS-DHP formed in incubations with Chinese and Caucasian liver microsomes at each substrate concentration are presented in the supplementary materials 5 . Since the 7-GS-DHP metabolite formed from lasiocarpine and riddelliine is the same, also bioactivation of the two compounds can be compared based on peak areas.

Table 2 Kinetic constants for depletion of lasiocarpine and riddelliine by Chinese and Caucasian tissue fractions

\begin{tabular}{|c|c|c|c|c|c|c|}
\hline \multirow[t]{2}{*}{ Organ } & \multicolumn{3}{|l|}{ Chinese } & \multicolumn{3}{|l|}{ Caucasian } \\
\hline & $\begin{array}{l}V_{\max }{ }^{\mathrm{a}}(\mathrm{nmol} / \mathrm{min} / \mathrm{mg} \\
\text { microsomal protein) }\end{array}$ & $K_{\mathrm{m}}^{\mathrm{a}}(\mu \mathrm{M})$ & $\begin{array}{l}\text { Catalytic efficiency }{ }^{\mathrm{b}}(\mu \mathrm{L} / \\
\mathrm{min} / \mathrm{mg} \text { microsomal } \\
\text { protein) }\end{array}$ & $\begin{array}{l}V_{\max }{ }^{\mathrm{a}}(\mathrm{nmol} / \mathrm{min} / \mathrm{mg} \\
\text { microsomal protein) }\end{array}$ & $K_{\mathrm{m}}^{\mathrm{a}}(\mu \mathrm{M})$ & $\begin{array}{l}\text { Catalytic efficiency }{ }^{\mathrm{b}}(\mu \mathrm{L} / \\
\mathrm{min} / \mathrm{mg} \text { microsomal } \\
\text { protein) }\end{array}$ \\
\hline \multicolumn{7}{|c|}{ Lasiocarpine } \\
\hline Liver & $4.6 \pm 0.9$ & $52.5 \pm 23.2$ & 87.8 & $5.1 \pm 0.9$ & $25.8 \pm 13.0$ & 197.7 \\
\hline Intestine & n.a. ${ }^{\mathrm{c}}$ & n.a. ${ }^{c}$ & - & $0.9 \pm 0.2$ & $72.1 \pm 37.9$ & 12.5 \\
\hline Lung & n.a. ${ }^{c}$ & n.a. ${ }^{c}$ & - & n.d. ${ }^{\mathrm{d}}$ & n.d. ${ }^{\mathrm{d}}$ & - \\
\hline Kidney & n.a. ${ }^{c}$ & n.a. ${ }^{c}$ & - & n.d. ${ }^{\mathrm{d}}$ & n.d. ${ }^{\mathrm{d}}$ & - \\
\hline \multicolumn{7}{|l|}{ Riddelliine } \\
\hline Liver & $0.3 \pm 0.05$ & $195.5 \pm 61.0$ & 1.6 & $2.0 \pm 0.5$ & $274.1 \pm 150.7$ & 7.3 \\
\hline Intestine & n.a. ${ }^{c}$ & n.a. ${ }^{\mathrm{c}}$ & - & $0.2 \pm 0.1$ & $380.4 \pm 285.3$ & 0.5 \\
\hline Lung & n.a. ${ }^{\mathrm{c}}$ & n.a. ${ }^{c}$ & - & n.d. ${ }^{\mathrm{d}}$ & n.d. ${ }^{\mathrm{d}}$ & - \\
\hline Kidney & n.a. ${ }^{c}$ & n.a. ${ }^{c}$ & - & n.d. ${ }^{\mathrm{d}}$ & n.d. ${ }^{\mathrm{d}}$ & - \\
\hline
\end{tabular}

${ }^{\mathrm{a}}$ Average $\pm \mathrm{SD}$ of three independent experiments

${ }^{\mathrm{b}} V_{\max } / K_{\mathrm{m}} \times 1000$

${ }^{\mathrm{c}}$ Not available

${ }^{\mathrm{d}}$ Not detected 
a

Lasiocarpine
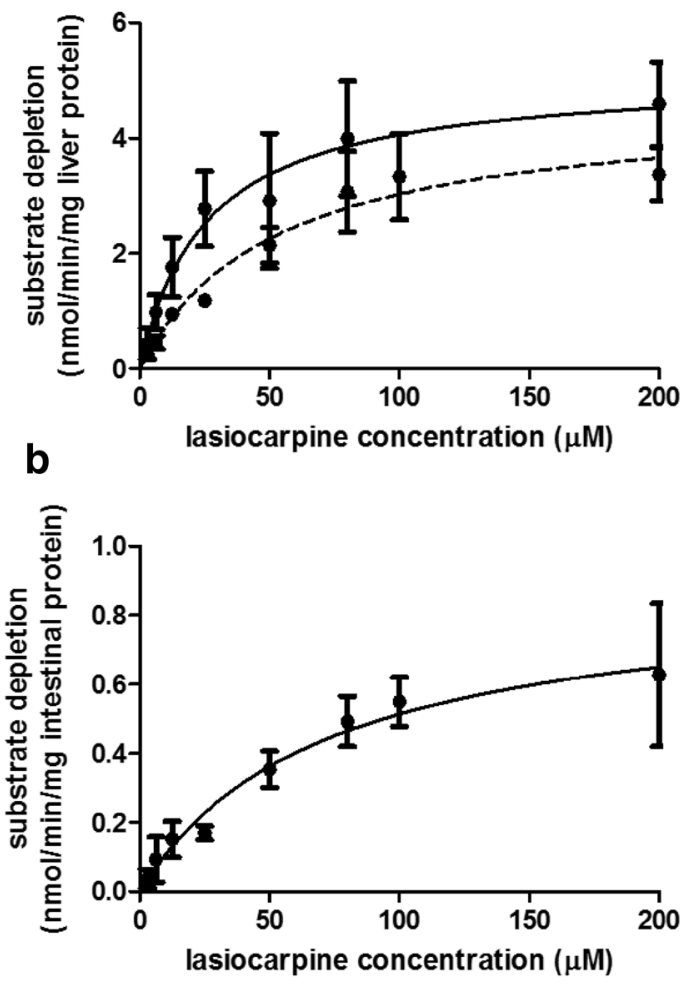

Fig. 4 Concentration-dependent rate of depletion of lasiocarpine and riddelliine in incubations with Chinese (dotted line) and Caucasian (solid line) liver microsomes (a) and Caucasian intestinal microsomes

\section{Comparison of the scaled kinetic constants for lasiocarpine and riddelliine by human and rat tissue fractions}

In a subsequent step the kinetic constants presented in Table 2 were scaled to human tissues. To this end, the in vitro $V_{\max }$ values expressed as $\mathrm{nmol} / \mathrm{min} / \mathrm{mg}$ microsomal protein (Table 2) were scaled to $\mu \mathrm{mol} / \mathrm{h} / \mathrm{kg}$ bw using the microsomal protein yields of $35 \mathrm{mg} /(\mathrm{g}$ liver $)$ or $20.6 \mathrm{mg} /(\mathrm{g}$ small intestine) as scaling factors as described previously (Cubitt et al. 2009; Medinsky et al. 1994), bodyweight and tissue fractions of human (Chinese and Caucasian) and rat (Chen et al. 2018). This scaling allows the comparison of the kinetic constants obtained from tissue fractions of different species and from different organs. Table 3 presents a summary of the kinetic parameters for lasiocarpine and riddelliine by human (Chinese and Caucasian) tissue fractions thus obtained and the comparable kinetic parameter values previously reported for rat tissue fractions (Chen et al. 2018).

Comparison of the kinetic constants between human (Chinese and Caucasians) and rat shows that liver is the major organ for lasiocarpine and riddelliine metabolism.

\section{Riddelliine}
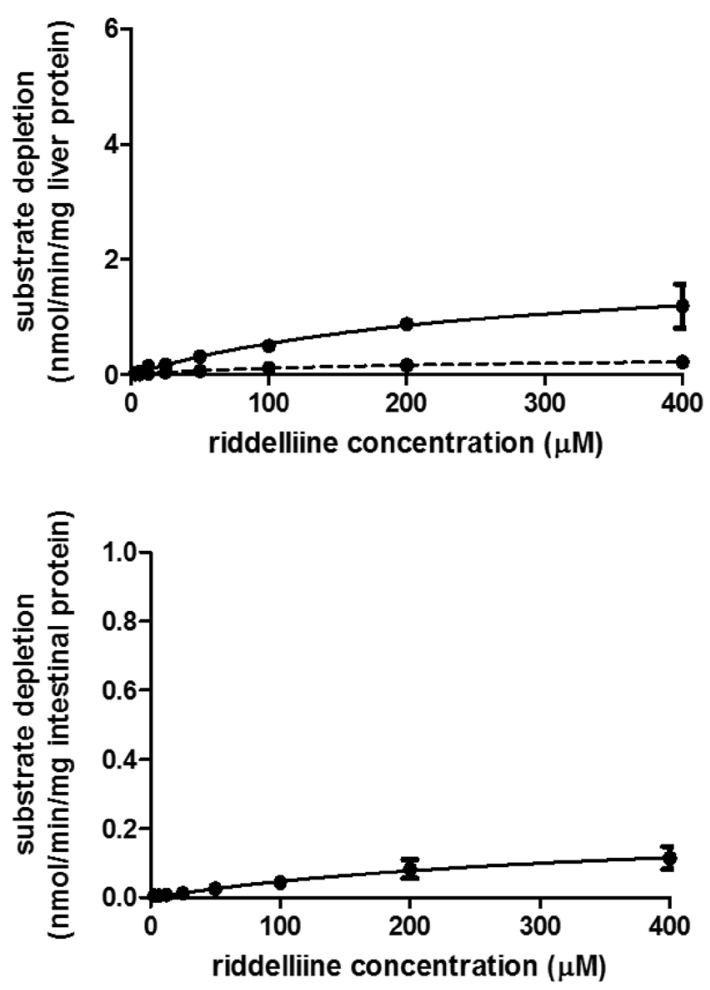

(b) in the presence of the cofactor NADPH. Data points represent mean values \pm SD of three individual experiments

Compared to the human (Caucasian), the contribution of intestinal metabolism for both compounds is higher in the rat. The catalytic efficiency for the depletion of lasiocarpine is generally 3.2-fold lower in human liver (Chinese and Caucasian) and 6-fold lower in human intestine (Caucasian) than in rat. The overall catalytic efficiency for depletion of lasiocarpine in human (Caucasian) is 1.9-fold lower than in rat. The catalytic efficiency for the depletion of riddelliine is generally 16.8-fold lower in human liver (Chinese and Caucasian) and 1.2-fold lower in small intestine (Caucasian) compared to those values in rat tissues. The overall catalytic efficiency for depletion of riddelliine in human (Caucasian) is 5.0-fold lower. Those generally lower values of catalytic efficiency for depletion of lasiocarpine and riddelliine in human liver and small intestine compared to the rat were mainly due to relatively higher $K_{\mathrm{m}}$ values for depletion of both compounds in human. 
Table 3 Scaled kinetic constants for depletion of lasiocarpine and riddelliine by human (Chinese and Caucasian) and rat tissue fractions

\begin{tabular}{|c|c|c|c|c|c|c|}
\hline \multirow[b]{2}{*}{ Organ } & \multicolumn{3}{|c|}{ Human (Chinese/Caucasian) } & \multicolumn{3}{|l|}{ Rat } \\
\hline & $\begin{array}{l}\text { Scaled } V_{\max }^{\mathrm{a}} \\
(\mu \mathrm{mol} / \mathrm{h} / \mathrm{kg} \mathrm{bw})\end{array}$ & $K_{\mathrm{m}}(\mu \mathrm{M})$ & $\begin{array}{l}\text { Scaled catalytic } \\
\text { efficiency }^{\mathrm{b}}(\mathrm{L} / \mathrm{h} / \mathrm{kg} \mathrm{bw})\end{array}$ & $\begin{array}{l}\text { Scaled } V_{\max }^{\mathrm{a}, \mathrm{c}} \\
(\mu \mathrm{mol} / \mathrm{h} / \mathrm{kg} \mathrm{bw})\end{array}$ & $K_{\mathrm{m}}^{\mathrm{a}, \mathrm{c}}(\mu \mathrm{M})$ & $\begin{array}{l}\text { Scaled catalytic } \\
\text { efficiency }{ }^{\mathrm{bc}}(\mathrm{L} / \mathrm{h} / \\
\mathrm{kg} \mathrm{bw})\end{array}$ \\
\hline \multicolumn{7}{|c|}{ Lasiocarpine } \\
\hline Liver & $222.2^{\mathrm{e}} / 278.2^{\mathrm{f}}$ & $52.5^{\mathrm{e}} / 25.8^{\mathrm{f}}$ & $4.2^{\mathrm{e}} / 10.8^{\mathrm{f}}$ & 377.4 & 19.5 & 19.4 \\
\hline Intestine & $\mathrm{n} . \mathrm{a}^{\mathrm{d}, \mathrm{e}} / 9.9^{\mathrm{f}}$ & $\mathrm{n} \cdot \mathrm{a}^{\mathrm{d}, \mathrm{e}} / 72.1^{\mathrm{f}}$ & n. $\mathrm{a}^{\mathrm{d}, \mathrm{e}} / 0.2^{\mathrm{f}}$ & 29.4 & 23.4 & 1.3 \\
\hline \multicolumn{7}{|l|}{ Riddelliine } \\
\hline Liver & $14.5^{\mathrm{e}} / 109.2^{\mathrm{f}}$ & $195.5^{\mathrm{e}} / 274.1^{\mathrm{f}}$ & $0.07^{\mathrm{e}} / 0.4^{\mathrm{f}}$ & 149.6 & 75.7 & 2.0 \\
\hline Intestine & $\mathrm{n} \cdot \mathrm{a}^{\mathrm{d}, \mathrm{e}} / 1.8^{\mathrm{f}}$ & $\mathrm{n} \cdot \mathrm{a}^{\mathrm{d}, \mathrm{e}} / 380.4^{\mathrm{f}}$ & $\mathrm{n} \cdot \mathrm{a}^{\mathrm{d}, \mathrm{e}} / 0.005^{\mathrm{f}}$ & 1.4 & 221.0 & 0.006 \\
\hline
\end{tabular}

${ }^{\text {a }}$ Scaled $V_{\max }$ calculated from the in vitro $V_{\max }$ using the scaling factor of microsome protein yields of $35 \mathrm{mg} /(\mathrm{g}$ liver) or $20.6 \mathrm{mg} /(\mathrm{g}$ small intestine), bodyweight and tissue fractions of human (Chinese and Caucasian) and rat

${ }^{\mathrm{b}}$ Scaled catalytic efficiency (scaled $V_{\max } / K_{\mathrm{m}}$ )

${ }^{\mathrm{c}}$ Chen et al. (2018)

${ }^{\mathrm{d}}$ Not available

${ }^{\text {e}}$ Scaled kinetic constants by Chinese tissue fractions

${ }^{\mathrm{f}}$ Scaled kinetic constants by Caucasian tissue fractions

\section{Unbound fraction in human blood as determined in the RED assay}

The unbound fraction for lasiocarpine in human plasma was determined to be 0.43 . For as yet unknown reasons, and in contrast to lasiocarpine, the unbound faction $\left(f_{\mathrm{ub}}\right)$ of riddelliine could not be detected after incubation in the RED device, even not when the concentration was increased from 5 to $50 \mu \mathrm{M}$. Previously, Chen et al. (2018) defined $f_{\mathrm{ub}}$ values for lasiocarpine and riddelliine by rat serum using the same method as provided in "Protein binding correction" section, and reported that the $f_{\mathrm{ub}}$ values of lasiocarpine and riddelliine in rat plasma were comparable, amounting to 0.64 for lasiocarpine and to 0.66 for riddelliine. Therefore, in the present study the $f_{\mathrm{ub}}$ of riddelliine in human plasma was assumed to be the same as the $f_{\mathrm{ub}}$ value of lasiocarpine in human plasma amounting to 0.43 .

\section{PBK model prediction and evaluation}

To evaluate the performance of the human PBK models, ideally the model outcomes should be compared to available human data. Since such human in vivo data on the kinetics of lasiocarpine and riddelliine are not available, model evaluation is mainly based on evaluation of the same model in our previous study where we developed and evaluated the model for riddelliine and lasiocarpine in rat and mouse (Chen et al. 2018). Chen et al. (2018) described the performance of the rat and mouse PBK model of riddelliine by comparing the predicted $C_{\max }$ of riddelliine in rat and mouse blood to the observed in vivo data reported in the literature (Chen et al. 2018). Data revealed that the predicted levels of
$C_{\max }$ of riddelliine in rat and mouse blood and the $C_{\max }$ values observed in these species in the in vivo studies matched adequately (Chen et al. 2018). An additional evaluation of the model emerged from comparison of the PBK modellingbased predicted $\mathrm{BMDL}_{5}-\mathrm{BMDU}_{5}$ values derived from the predicted in vivo dose-response curve for liver toxicity of lasiocarpine in rats. The results fell well within the range of PoDs obtained from actual in vivo studies on acute liver toxicity of lasiocarpine in rats, indicating that the PoDs derived from the combined in vitro and in silico PBK modelling approach provided a good approximation of in vivo acute liver toxicity of lasiocarpine (Chen et al. 2018). Based on these results for the rat model, the developed PBK model for lasiocarpine or riddelliine are considered an adequate first approximation to describe the in vivo situation for human. An additional approach to describe the performance of the human PBK model for lasiocarpine and riddelliine for the human populations, especially for the difference between the Chinese and the Caucasian populations, was done based on the approach described previously (Ning et al. 2017). In this approach a comparison is made between the predicted inter-ethnic differences for the dose-dependent concentration of lasiocarpine and riddelliine in the liver blood and the observed inter-ethnic differences in hepatic metabolising enzymes that mainly catalyse the depletion of lasiocarpine or riddelliine. CYP 3A4 predominately catalyses the depletion of lasiocarpine and riddelliine in human liver (Fu et al. 2004; Miranda et al. 1991; Wiedenfeld and Edgar 2011). Figure 5 shows that at similar dose levels the predicted $C_{\max }$ of lasiocarpine and riddelliine in the liver blood from average Chinese subjects is generally 2- to 3-fold higher than in the liver from average Caucasian subjects. This is mainly 

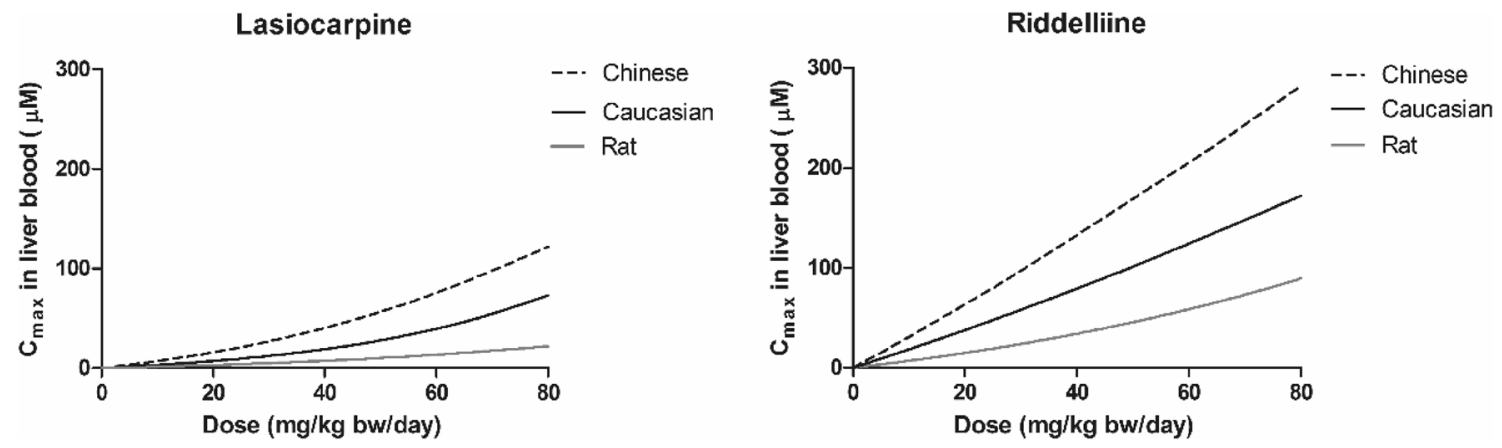

Fig. 5 PBK modelling-based predictions for the dose-dependent $C_{\max }$ of lasiocarpine and riddelliine in liver blood in Chinese (black dotted line), Caucasian (black solid line) and rat (grey line)

due to the fact that the catalytic efficiency for depletion of lasiocarpine and riddelliine by Chinese liver microsomes is 2- to 4-fold lower compared to Caucasian liver microsomes. The prediction of higher $C_{\max }$ values for lasiocarpine and riddelliine for Chinese subjects is in line with the reported data for the $C_{\max }$ and AUC of alprazolam, the substrate for CYP 3A 4/5, for which the $C_{\max }$ and AUC for Asian subjects (ten Chinese, three Filipino, and one Japanese) were 1.2and 1.3-fold, respectively, higher than for Caucasian subjects $(n=14)$ (Lin et al. 1988). Furthermore, in a meta-analysis Barter et al. (2013) showed that the plasma clearance of the CYP 3A4/5 substrate midazolam following an oral dosing was 2.4-fold higher in Caucasian than in Chinese subjects.

For the inter-species comparison, the predicted $C_{\max }$ of lasiocarpine and riddelliine in the human (Chinese and Caucasian) liver blood is generally 2 - to 5 -fold higher than those in the rat liver blood as shown in Fig. 5. This is because the catalytic efficiency for depletion of both compounds in human (Caucasian) is 2- to 5-fold lower compared to rat. Altogether, the PBK models obtained in the present study were considered adequate for further prediction of the interspecies and inter-ethnic differences in hepatotoxicity of lasiocarpine and riddelliine.

\section{Sensitivity analysis}

The normalized sensitivity coefficients of the model parameters for prediction of the $C_{\max }$ of lasiocarpine and riddelliine in liver blood of Chinese and Caucasian was analyzed. The sensitivity coefficients were calculated at a dose of $8 \mathrm{ng} /$ $\mathrm{kg}$ bw and $3 \mathrm{mg} / \mathrm{kg}$ bw lasiocarpine and riddelliine and all model parameters with sensitivity coefficients higher than $10.1 \mid$ are shown in the supplementary materials 6 . The results obtained reveal that for both Chinese and Caucasian subjects at a dose of $8 \mathrm{ng} / \mathrm{kg}$ bw lasiocarpine the predicted $C_{\max }$ in liver blood in the PBK model is most sensitive to liverrelated parameters and the absorption rate constant $(\mathrm{Ka})$ for uptake from the GI tract. At $3 \mathrm{mg} / \mathrm{kg}$ bw lasiocarpine, the results of the sensitivity analysis of both ethnic groups are similar to the situation at $8 \mathrm{ng} / \mathrm{kg}$ bw lasiocarpine. The results for the sensitivity analysis of the PBK models for riddelliine shown in Figure S6 reveal similar results showing that $\mathrm{Ka}$ and liver-related parameters have the highest influence on the predicted $C_{\max }$ of riddelliine in the liver blood in both ethnic groups. At $8 \mathrm{ng} / \mathrm{kg}$ bw riddelliine the results of the sensitivity analysis of both ethnic groups are similar to the analysis at $3 \mathrm{mg} / \mathrm{kg}$ bw. For both compounds, Ka and liver-related parameters have less influence on the predicted $C_{\max }$ in the liver blood in average Chinese compared to average Caucasian.

\section{Predicted inter-ethnic differences in hepatotoxicity of lasiocarpine and riddelliine}

Figure 6 shows the predicted in vivo dose-response curves for liver toxicity of lasiocarpine and riddelliine in Chinese and Caucasian subjects obtained by translation of the in vitro concentration-response curves obtained from human hepatocytes (Fig. 3) by reverse dosimetry using the respective PBK models. The response $\%$ indicated at the y-axis of the in vivo dose-response curve was calculated based on Eq. (4): response $\%=100 \%$ (solvent control)—cell viability $\%$ in the in vitro concentration-response curve.

Table 4 presents the $\mathrm{BMDL}_{5}-\mathrm{BMDU}_{5}$ values derived from the predicted dose-response curves as shown in Fig. 6 for Chinese and Caucasian subjects. The values of the $\mathrm{BMD}_{5}$ (benchmark dose corresponding to 5\% extra risk) were also presented to enable calculation of the ratios between the $\mathrm{BMD}_{5}$ and $\mathrm{BMDL}_{5}$ or $\mathrm{BMDU}_{5}$ showing they are generally lower than 3, which was one of the criteria for acceptance of the model fit. Detailed information on the BMD analysis can be found in the supplementary materials 7 .

Interestingly, the PBK modelling-based predictions for in vivo human toxicity of riddelliine could be evaluated using available human data, supporting further evaluation of the PBK models and the PBK modelling-based reverse dosimetry 


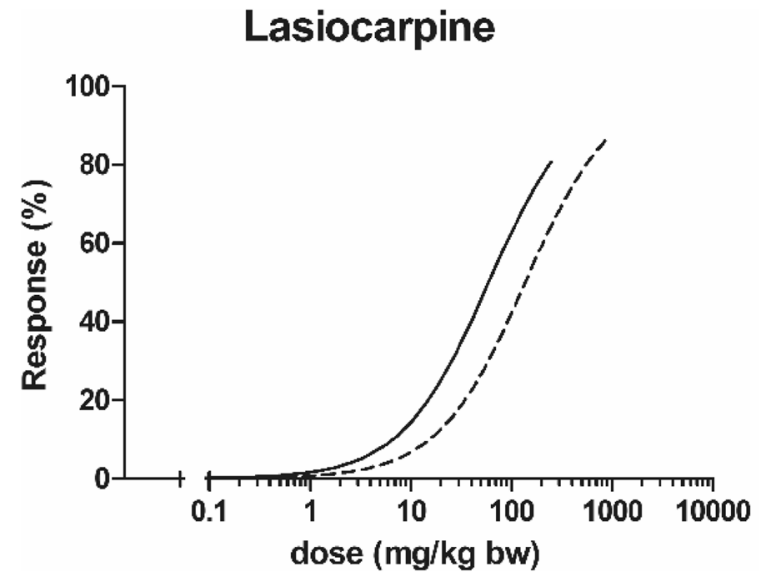

Fig. 6 Predicted dose-response curves for acute liver toxicity of lasiocarpine and riddelliine in average Chinese (black dotted line) and average Caucasians (black solid line) as obtained by translation of

Table 4 Predicted $\mathrm{BMD}_{5}$ and $\mathrm{BMDL}_{5}-\mathrm{BMDU}_{5}$ values derived from dose-response curves predicted using PBK modelling-based reverse dosimetry of in vitro cytotoxicity data in human hepatocytes

\begin{tabular}{lll}
\hline Compounds & \multicolumn{2}{l}{$\begin{array}{l}\text { Predicted } \mathrm{BMDL}_{5}-\mathrm{BMDU}_{5}\left(\mathrm{BMD}_{5}\right)(\mathrm{mg} / \\
\mathrm{kg} \text { bw/day })\end{array}$} \\
\cline { 2 - 3 } & Chinese & Caucasian \\
\hline Lasiocarpine & $14.7-41.2(26.0)$ & $7.4-23.7(14.1)$ \\
Riddelliine & $1.0-5.9(2.6)$ & $0.2-1.2(0.5)$ \\
\hline
\end{tabular}

approach. The study of Culvenor (1983) estimated that in human subjects a dose of $1.4-3 \mathrm{mg} / \mathrm{kg}$ bw/day and $0.7-1.5 \mathrm{mg} /$ $\mathrm{kg}$ bw/day of riddelliine may lead to liver necrosis (exposure of a boy for shorter than 2 weeks) and liver cirrhosis (exposure of a girl for 2 weeks), respectively (Culvenor 1983). The BMD values for Caucasian and Chinese subjects predicted in the present study are comparable since they amount to 0.5 and $2.6 \mathrm{mg} / \mathrm{kg}$ bw/day, respectively. For the inter-ethnic comparison, the predicted $\mathrm{BMDL}_{5}$ of lasiocarpine was observed to be 2.0-fold higher for Chinese than for Caucasian subjects and for riddelliine the predicted $\mathrm{BMDL}_{5}$ value was 5.0-fold higher for Chinese than for Caucasian subjects, indicating Chinese may be less sensitive to the liver toxicity of these PAs than the Caucasian population. This difference can be mainly ascribed to the lower relative level of bioactivation resulting in lower toxicity at similar dose levels in spite of the slower metabolic clearance.

\section{Riddelliine}

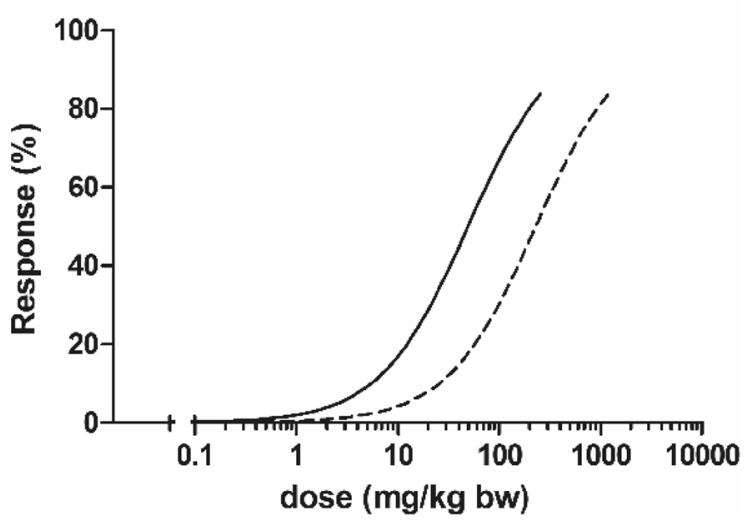

cytotoxicity concentration-response data obtained with human hepatocytes using PBK modelling-based reverse dosimetry

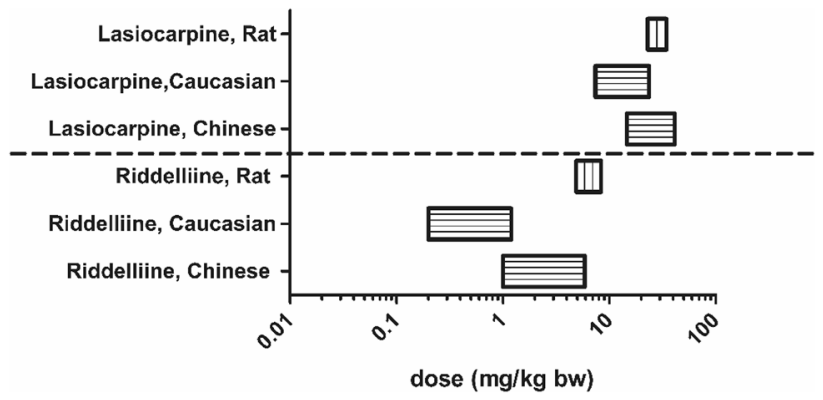

Fig. 7 Predicted $\mathrm{BMDL}_{5}-\mathrm{BMDU}_{5}$ values for acute liver toxicity of lasiocarpine (above the dashed line) and riddelliine (below the dashed line) in humans (Caucasians and Chinese) (bars containing horizontal lines) predicted by the PBK modelling-based reversed dosimetry approach using data for human hepatocytes from the present study as compared to the predicted $\mathrm{BMDL}_{5}-\mathrm{BMDU}_{5}$ values for acute liver toxicity of lasiocarpine (above the dashed line) and riddelliine (below the dashed line) in rat (bars containing vertical lines) derived from our previous study using data for rat hepatocytes and a rat PBK model (Chen et al. 2018)

\section{Predicted species differences in hepatotoxicity of lasiocarpine and riddelliine}

To obtain insight in possible species differences in sensitivity toward liver toxicity of the PAs, the $\mathrm{BMDL}_{5}-\mathrm{BMDU}_{5}$ values of lasiocarpine and riddelliine for acute liver toxicity for humans (Chinese and Caucasians) (Table 4) defined in the present study were compared to the same PoDs defined previously for rats using in vitro toxicity data in rat hepatocytes and PBK models for rats (Chen et al. 2018) (see Fig. 7). The $\mathrm{BMDL}_{5}-\mathrm{BMDU}_{5}$ values derived from the predicted dose-response curves for lasiocarpine for humans amounting to $11.1-32.3 \mathrm{mg} / \mathrm{kg}$ bw (average PoDs of Chinese and Caucasians) are up to 2.0 -fold lower than the predicted 
$\mathrm{BMDL}_{5}-\mathrm{BMDU}_{5}$ values of Chen et al. (2018) for lasiocarpine for rat which amounted to $23.0-34.4 \mathrm{mg} / \mathrm{kg}$ bw/day. For riddelliine, the predicted $\mathrm{BMDL}_{5}-\mathrm{BMDU}_{5}$ values for humans amounting to $0.6-3.6 \mathrm{mg} / \mathrm{kg}$ bw (average PoDs of Chinese and Caucasians) are up to 8.2-fold lower than the $\mathrm{BMDL}_{5}-\mathrm{BMDU}_{5}$ for rat amounting to $4.9-8.4 \mathrm{mg} / \mathrm{kg}$ bw/ day. Thus, humans seem to be somewhat more sensitive to liver toxicity of these PAs than rats.

\section{Discussion}

The aim of the present study was to use PBK modellingbased reverse dosimetry to translate human cytotoxicity data of lasiocarpine and riddelliine to in vivo dose-response data in humans (Chinese and Caucasians) and study ethnic human differences. Comparison of the results to a similar study previously performed for rats (Chen et al. 2018) would provide insight in species differences in sensitivity to these two PAs.

The PBK model developed in the present study describes the kinetics of lasiocarpine and riddelliine and not of their metabolites, since we assume that the acute liver toxicity of lasiocarpine and riddelliine can be modelled based on cytotoxicity data of the parent compound provided that the in vitro model used contains the adequate enzymes required for their bioactivation to hepatotoxic metabolites. Especially the dehydropyrrolizidine alkaloid metabolites of PAs are reactive with proteins and DNA, thereby causing toxic effects (Fu et al. 2004). The in vitro cytotoxicity experiment using HepaRG cells and human hepatocytes can be expected to take this bioactivation into account. The HepaRG cell line is a human hepatoma cellular model consisting of a mixture of both hepatocyte-like and biliary-like cells (Lambert et al. 2009). After the treatment with DMSO, HepaRG cells differentiate into hepatocyte-like morphology. Kanebratt and Andersson (2008) reported that the expression of P450 in HepaRG cells was generally lower compared with human hepatocytes, except for a higher expression of CYP 3A4 and CYP 7A1. However, the CYP 3A4 activity was still about 1.5 -fold higher in human hepatocytes in 2 out of 3 individuals compared to DMSO treated HepaRG cells as determined by measuring the clearance of the CYP $3 \mathrm{~A} 4$ probe substrate midazolam (Kanebratt and Andersson 2008). Other studies also showed that P450 activities such as CYP 1A2, CYP 2B6, CYP 2C8, CYP 2C9, CYP 2C19 and CYP 2D6 were generally lower in HepaRG cells than in human hepatocytes, except for CYP $3 \mathrm{~A} 4$ showing a generally 1.5 -fold higher activity in HepaRG cells (Kvist et al. 2018; Lubberstedt et al. 2011). However, Gerets et al. (2012) reported that CYP 3A4 activity was about 17.0-fold lower in the HepaRG cells than in freshly isolated human hepatocytes from three different donors. This conflicting result may result from inter-individual variability in CYP 3A4 activity and/or from differences in the level of enzyme induction in the DMSO treated HepaRG cells. However, based on the limited difference in the cytotoxicity of riddelliine and lasiocarpine in primary hepatocytes and the HepaRG cells observed in the present study (Fig. 3), we conclude that the induction of the CYPs involved in bioactivation of these PAs in the HepRG cells was adequate. Gerets et al. (2012) also reported that cryopreserved primary human hepatocytes showed a 3-fold higher sensitivity for the detection of hepatotoxic compounds compared with HepaRG cells. In the present study, the $\mathrm{IC}_{50}$ value for lasiocarpine and riddelliine derived from HepaRG cells was 10- and 3-fold higher, respectively, than the $\mathrm{IC}_{50}$ derived from studies with human hepatocytes, probably reflecting the lower metabolic capacity of the HepaRG cells for bioactivation of the PAs. Given these differences, prediction of the acute liver toxicity of both PAs was based on the in vitro concentration-response curves obtained with human primary hepatocytes.

Based on the sensitivity analysis, the predicted $C_{\max }$ values of lasiocarpine and riddelliine in average Chinese and average Caucasians are highly affected by the absorption rate constant $(\mathrm{Ka})$ for uptake from the GI tract and the kinetic constants for metabolic clearance of the parent compounds in the liver. Variability in the kinetic constants for metabolic clearance can result from the individual differences in the expression of CYP 3A4 which is the primary enzyme for metabolizing lasiocarpine and riddelliine in humans (Miranda et al. 1991). The literature reports that the abundance of CYP 3A4 in the liver varies significantly in individuals (Fu et al. 2004), and the inter-individual variation in the level of CYP 3A4 expression can vary up to 100-fold (Westlind-Johnsson et al. 2003). In addition to the kinetic constants, the Ka value also has a large influence on the model predictions. In the present study, the Ka values of lasiocarpine and riddelliine were $1.55 / \mathrm{h}$ and $0.72 / \mathrm{h}$, respectively as reported by Chen et al. (2018). In the study of Chen et al. (2018), Ka values of both compounds were defined based on $P_{\text {app }}$ values derived by two methods: Qikprop software (SchrOdinger, trial version, Germany) and a QSAR method developed by Hou et al. (2004). The $P_{\text {app }}$ values obtained from these two methods differ only 1.3fold for lasiocarpine, but 4.5-fold for riddelliine (Chen et al. 2018). The large difference in $P_{\text {app }}$ values for riddelliine may, therefore, largely affect PBK model-predicted internal concentrations and related predicted dose levels that cause acute toxicity. Previously, Chen et al. (2018) reported the predicted $\mathrm{BMDL}_{5}-\mathrm{BMDU}_{5}$ for riddelliine in rat amounting to $1.0-2.2 \mathrm{mg} / \mathrm{kg}$ bw/day when using the $\mathrm{Ka}(0.27 / \mathrm{h})$ obtained by Qikprop software and to 3.3-14.6 mg/kg bw/day when using the $\mathrm{Ka}(1.17 / \mathrm{h})$ obtained by the QSAR method of Hou et al. (2004). The PoDs for riddelliine obtained in rats when using the average $\mathrm{Ka}$ amount to $4.9-8.4 \mathrm{mg} / \mathrm{kg}$ 
bw/day (Chen et al. 2018). This outcome supports the outcome of the sensitivity analysis indicating that the Ka value has a large influence on the model predictions. This leads to the conclusion that further refinement of especially the $\mathrm{P}_{\text {app }}$ and resulting Ka value may improve the models and their predictions. However, given that the predicted $\mathrm{BMD}_{5}$ values for riddelliine in Chinese and Caucasian subjects amounting to 2.6 and $0.5 \mathrm{mg} / \mathrm{kg}$ bw/day, respectively are comparable to the literature reported in vivo data for human subjects amounting to $0.7-3 \mathrm{mg} / \mathrm{kg}$ bw/day (Culvenor 1983) when using the average Ka values, the use of the average Ka value for riddelliine seems a reasonable strategy. A recent study reported that the pyrrolizidine alkaloid monocrotaline (retronecine-type PA) is a high-affinity substrate of the organic cation transporter 1 (OCT1), a transporter mainly expressed in liver and involved in uptake of chemicals from blood into the hepatocytes. This study suggested that active transport mechanisms may be involved in the cellular uptake of pyrrolizidine alkaloids into the liver tissue (Tu et al. 2013). In the present study, we used $\log K_{\text {ow }}$ to estimate the liver tissue:blood partition coefficients of lasiocarpine and riddelliine. In perfusion-limited PBK models, such as used in our study, an immediate partitioning between plasma and tissue is assumed, which is not dependent on chemical diffusion across cell membranes and/or active uptake processes. Therefore, we do not expect to underestimate hepatic concentrations in our models. Given that the sensitivity analysis revealed that the $C_{\max }$ of lasiocarpine and riddelliine in liver blood were not highly affected by the liver tissue:blood partition coefficients (see supplementary materials 6), estimation of this kinetic parameter for uptake of lasiocarpine and riddelliine into liver tissue based on partitioning between plasma and tissue is unlikely to affect the outcomes to a significant extent.

Given that the methods used in the present study and in the previous study (Chen et al. 2018) for predicting the in vivo liver toxicity of lasiocarpine and riddelliine in rat were similar, using species-specific primary hepatocytes and species-specific subcellular tissue fractions in similar in vitro incubations to define the species-specific metabolic clearance, using a similar PBK model structure, the model predictions also provide insight in species differences in acute liver toxicity of lasiocarpine and riddelliine. This inter-species comparison, revealed that the predicted $\mathrm{BMDL}_{5}$ for liver toxicity of lasiocarpine and riddelliine in humans (average PoDs of Chinese and Caucasians) is up to 2.0-fold and 8.2-fold lower, respectively, than that for rat, indicating that humans are somewhat more sensitive towards acute liver toxicity of lasiocarpine and riddelliine than rats. It is of interest to note that the in vitro cytotoxicity of these two compounds in human Caucasian hepatocytes was lower than in rat hepatocytes, and that the predicted higher toxicity in vivo thus results from inter-species differences in the kinetics. The kinetics showed a 1.9- and 5.0-fold slower clearance of lasiocarpine and riddelliine in humans (Caucasian) compared to rats. According to literature, the metabolism of PAs in rodents is mainly catalyzed by CYP $2 \mathrm{~B}$ and CYP 3A subfamilies, while in humans, metabolism is mainly catalysed by CYP 3A4 (Li et al. 2011; Miranda et al. 1991). This observation is of interest when considering rat toxicity data for human risk assessment. Extrapolating rodent toxicity data to the human situation usually includes the use of a default uncertainty factor of 10 to account for species differences, consisting of a factor of 4.0 for kinetic and a factor of 2.5 for dynamic differences (WHO 1999). In the present study, the inter-species differences in acute liver toxicity of lasiocarpine and riddelliine fall well within the default factor of 10 . Such a compound-specific uncertainty factor that could be smaller than the default value of 10 was also reported for aristolochic acid I for which in vitro-PBK model facilitated reverse dosimetry-based predicted PoDs differed about 1.8 -fold between rat and human and about 1.9-fold between mouse and human (Abdullah et al. 2016). Also for the bioactivation of estragole the compound specific uncertainty factor for differences in kinetics between humans and rats was predicted to be 2-fold and thus smaller than the default factor of 4.0 for kinetic differences (Punt et al. 2009). Altogether, these results reveal that the in vitro-PBK modelling-based reverse dosimetry may provide a way forward to define compound specific uncertainty factors, also called compound specific adjustment factors (CSAFs), in risk assessment.

Comparison of the predicted $\mathrm{BMDL}_{5}$ for lasiocarpine and riddelliine between Chinese and Caucasian subjects showed that for Chinese subjects, the predicted $\mathrm{BMDL}_{5}$ for lasiocarpine was 2.0-fold higher and for riddelliine 5.0-fold higher than for Caucasians, suggesting that Chinese subjects might be less sensitive towards acute hepatotoxicity of lasiocarpine and riddelliine. This is mainly due to the differences in bioactivation of the parent compound, resulting in less bioactivation for both compounds at similar dose levels in average Chinese compared to average Caucasian subjects, and occurs in spite of less efficient clearance in Chinese as compared to Caucasians. In the present study, human hepatocytes used to study the in vitro cytotoxicity of lasiocarpine and riddelliine were derived from Caucasian subjects. Since human hepatocytes derived from Chinese subjects are not available, in vitro cytotoxicity for Chinese hepatocytes was defined by read-across from the Caucasian liver hepatocytes taking into account the difference in bioactivation between Chinese and Caucasian liver microsomes, as reflected by 7-GS-DHP formation in in vitro incubations. In the present study, we used GSH as a trapping agent to estimate the amount of reactive metabolites formed in liver microsomal incubations which is in line with the method described previously (Tamta et al. 2012). It has been reported that dehydro-PAs react with 
GSH forming there metabolites, 7-GS-DHP, 9-GS-DHP and 7,9-di-GSH-DHP, of which 7-GS-DHP is the major metabolite formed in vivo and in vitro (Chen et al. 2016; Lame et al. 1990; Lin et al. 1998; Reed et al. 1992; Tamta et al. 2012), whereas 7,9-di-GS-DHP is only formed in vitro (Lin et al. 1998; Reed et al. 1992; Tamta et al. 2012). Other factors influencing the dynamics of liver toxicity caused by PAs, for example, the relative potential for repair, cannot be taken into account in this way. Nevertheless, the approach now taken provides an adequate first approach to study the potential ethnic differences in the toxicokinetic process of PA-induced liver toxicity.

The ethnic differences in metabolic clearance of lasiocarpine and riddelliine determined by substrate depletion using liver microsomess for both populations was 2- to 4-fold, with clearance by the average Caucasian being more efficient. The difference in efficiency for formation of bioactive metabolites for both compounds by the Chinese and Caucasian incubations amounted to 7- to 8-fold, the value for the average Caucasian being highest. It has been reported that besides CYP 450 enzymes, flavin-containing monooxygenases (FMO) and carboxylesterases are considered to be involved in detoxification pathways as shown in Fig. 1 (Fu et al. 2004). FMO was found to be partly involved in the $\mathrm{N}$-oxide formation and carboxylesterases are involved in hydrolysis of the ester groups of PAs leading to the formation of necine base and necic acid moieties, which is considered to be the major detoxification pathway (Fu et al. 2004). Currently, two predominant carboxylesterases (CES) enzymes have been identified in human, i.e. CES 1 and CES 2 (Wang et al. 2018). A recent review reported that ethnic differences in carboxylesterases enzymes activity may be present due to single nucleotide polymorphisms (SNPs). For instance, CES 1 variants G1420E and D260fs, which are two important functional SNPs in Caucasian populations, showed reduced esterase activity in vitro, while these two variants were not found in the Asian populations (Cha et al. 2014; Wang et al. 2018). However, detailed studies of assessing ethnic differences in carboxylesterases activity are still lacking.

It is also of interest to note that in toxicological risk assessment, a default uncertainty factor of 10 is used to account for inter-individual variation, which can be divided into a factor 3.16 for kinetic and 3.16 for dynamic differences (WHO 1999). The ethnic human differences in predicted PoDs of lasiocarpine and riddelliine between Chinese and Caucasian subjects, were estimated to be 1.7 -and 5.0-fold respectively, and are thus in line with the default value of 3.16 for inter-individual kinetic differences. However, various studies report much higher than 2- to 5-fold inter-individual variability in the expression of the major hepatic CYP enzyme (CYP 3A4) involved in PA metabolism, ranging up to 100-fold (Westlind-Johnsson et al. 2003), or even up to 400-fold when taking illness, inhibition, and induction-related interactions into account (Galetin et al. 2004; Wilkinson 2005). Another study reported that interindividual variation in the expression of CYP $3 \mathrm{~A} 4$ varies 40-50-fold (Ingelman-Sundberg 2004). Therefore, the human inter-individual variation in kinetics is likely to be larger than the default value of 3.16. In a next step, to actually quantify inter-individual human variation in sensitivity to acute liver toxicity of lasiocarpine and riddelliine, the PBK model of the present study should be developed for individual subjects and combined with Monte Carlo modelling to predict hepatotoxicity within the human population. This Monte Carlo modelling should especially consider the variability in the PBK model parameters shown in the sensitivity analysis to influence the model predictions to the largest extent including the $\mathrm{Ka}$ and the kinetic parameters for metabolic clearance in the liver.

Given that the ultimate critical effect of lasiocarpine and riddelliine is not only acute hepatotoxicity but also genotoxicity and subsequently, carcinogenic transformation, it may also be of interest to extent the current PBK model to a physiologically based dynamic (PBD) model able to predict in vivo DNA binding as previously done for estragole (Paini et al. 2010). This would require the definition of an in vitro concentration response curve for DNA adduct formation in the primary hepatocytes, which can subsequently be translated to an in vivo dose-response curve for DNA adduct formation. According to the study of Xia et al. (2013), DNA adduct formation is a common biological biomarker of PAinduced tumorigenicity in rats. Previous studies reported that the metabolism of riddelliine by human liver microsomes resulted in a similar metabolic pattern and DNA adduct profile to those formed in the rat liver, suggesting that the mode of action of PAs studied in experimental rodents is highly relevant to humans (Xia et al. 2003; Zhao et al. 2012). The current PBK model could also translate in vitro concentration-response curves for DNA adduct formation in human liver cells to in vivo dose-response curves for DNA adduct formation in the liver of human.

In conclusion, the present study shows that PBK modelling-based reverse dosimetry can identify the differences in inter-species and inter-ethnic human differences in liver toxicity of lasiocarpine and riddelliine. The species-dependent variation in hepatotoxicity defined by the $\mathrm{BMDL}_{5}$ (average PoDs of Caucasians and Chinese) is up to 2.0-fold for lasiocarpine and 8.2-fold for riddelliine, with humans being more susceptible to lasiocarpine and riddelliine-induced liver toxicity than rat. The inter-ethnic human difference between average Chinese and average Caucasian subjects was estimated to be 2.0-fold for lasiocarpine and 5.0-fold for riddelliine, with the average Caucasian being more sensitive towards the acute liver toxicity of lasiocarpine and riddelliine, mainly due to more efficient reactive metabolite 
formation. Altogether, the present study shows proof-ofprinciple for a method to predict inter-species and interethnic differences in in vivo liver toxicity for PAs by an alternative testing strategy integrating in vitro cytotoxicity assays with PBK modelling-based reverse dosimetry.

Acknowledgements This work was funded by a grant from the China Scholarship Council (no. 201507720019 to NING JIA and no. 201508650023 to CHEN LU).

\section{Compliance with ethical standards}

Conflict of interest The authors declare that they have no conflict of interest.

OpenAccess This article is distributed under the terms of the Creative Commons Attribution 4.0 International License (http://creativeco mmons.org/licenses/by/4.0/), which permits unrestricted use, distribution, and reproduction in any medium, provided you give appropriate credit to the original author(s) and the source, provide a link to the Creative Commons license, and indicate if changes were made.

\section{References}

Abdullah R, Alhusainy W, Woutersen J, Rietjens IMCM, Punt A (2016) Predicting points of departure for risk assessment based on in vitro cytotoxicity data and physiologically based kinetic (PBK) modeling: The case of kidney toxicity induced by aristolochic acid I. Food Chem Toxicol 92:104-116

Adams IR, Hamlin KE, Jelinek CF, Phillips RF (1942) Structure of riddelliine, the alkaloid in Senecio riddellii. J Am Chem Soc 64:2760-2763

Bane A, Seboxa T, Mesfin G, Ali A, Tsegaye Y, M WT, Haile SGS T (2012) An outbreak of veno-occlusive liver disease in northern Ethiopia, clinical findings. Ethiop Med J 50(Suppl 2):9-16

Barter ZE, Tucker GT, Rowland-Yeo K (2013) Differences in Cytochrome P450-Mediated Pharmacokinetics Between Chinese and Caucasian Populations Predicted by Mechanistic Physiologically Based Pharmacokinetic Modelling. Clin Pharmacokinet 52(12):1085-1100

Bfr (2013) Pyrrolizidine alkaloids in herbal teas and teas. BfR opinion

Brown RP, Delp MD, Lindstedt SL, Rhomberg LR, Beliles RP (1997) Physiological parameter values for physiologically based pharmacokinetic models. Toxicol Ind Health 13(4):407-484

Cha Y-J, Jeong H-E, Shin J-G, Kim E-Y, Yu K-S, Cho J-Y, Hyun Yoon S, Lim K (2014) Genetic Polymorphisms of the Carboxylesterase 1 (CES1) Gene in a Korean Population. Transl Clin Pharmacol 22(2):30-34

Chan PC, Haseman JK, Prejean JD, Nyska A (2003) Toxicity and carcinogenicity of riddelliine in rats and mice. Toxicol Lett 144(3):295-311

Chan SC, Liu CL, Lo CM, Lam BK, Lee EW, Wong Y, Fan ST (2006) Estimating liver weight of adults by body weight and gender. World J Gastroenterol 12(14):2217-2222

Chen M, Li L, Zhong D, Shen S, Zheng J, Chen X (2016) 9-Glutathionyl-6,7-dihydro-1-hydroxymethyl-5H-pyrrolizine is the major pyrrolic glutathione conjugate of retronecine-type pyrrolizidine alkaloids in liver microsomes and in rats. Chem Res Toxicol 29(2):180-189

Chen L, Ning J, Louisse J, Wesseling S, Rietjens IMCM (2018) Use of physiologically based kinetic modelling-facilitated reverse dosimetry to convert in vitro cytotoxicity data to predicted in vivo liver toxicity of lasiocarpine and riddelliine in rat. Food Chem Toxicol 116(Pt B):216-226

Cubitt HE, Houston JB, Galetin A (2009) Relative importance of intestinal and hepatic glucuronidation-impact on the prediction of drug clearance. Pharm Res 26(5):1073-1083

Culvenor CC (1983) Estimated intakes of pyrrolizidine alkaloids by humans. A comparison with dose rates causing tumors in rats. J Toxicol Environ Health 11(4-6):625-635

DeJongh J, Verhaar HJM, Hermens JLM (1997) A quantitative property-property relationship (QPPR) approach to estimate in vitro tissue-blood partition coefficients of organic chemicals in rats and humans. Arch Toxicol 72(1):17-25

EFSA (2011) Scientific opinion on pyrrolizidine alkaloids in food and feed. EFSA Journal 9(11)

EFSA (2017) Risks for human health related to the presence of pyrrolizidine alkaloids in honey, tea, herbal infusions and food supplements. EFSA J 15(7)

EPA (2017) U.S. EPA Benchmark dose modeling guidance In. https:// www.efsa.europa.eu/sites/default/files/170301-0-p7.pdf

Evans MV, Andersen ME (2000) Sensitivity analysis of a physiological model for 2,3,7,8-tetrachlorodibenzo-p-dioxin (TCDD): assessing the impact of specific model parameters on sequestration in liver and fat in the rat. Toxicol Sci 54(1):71-80

Fashe MM, Juvonen RO, Petsalo A, Rasanen J, Pasanen M (2015) Species-Specific Differences in the in Vitro Metabolism of Lasiocarpine. Chem Res Toxicol 28(10):2034-2044

Field RA, Stegelmeier BL, Colegate SM, Brown AW, Green BT (2015) An in vitro comparison of the cytotoxic potential of selected dehydropyrrolizidine alkaloids and some N-oxides. Toxicon 97:36-45

Fu PP, Xia Q, Lin G, Chou MW (2004) Pyrrolizidine AlkaloidsGenotoxicity, Metabolism Enzymes, Metabolic Activation, and Mechanisms. Drug Metab Rev 36(1):1-55

Galetin A, Brown C, Hallifax D, Ito K, Houston JB (2004) Utility of recombinant enzyme kinetics in prediction of human clearance: Impact of variability, CYP3A5, and CYP2C19 on CYP3A4 probe substrates. Drug Metab Dispos 32(12):1411-1420

Gerets HHJ, Tilmant K, Gerin B, Chanteux H, Depelchin BO, Dhalluin S, Atienzar FA (2012) Characterization of primary human hepatocytes, HepG2 cells, and HepaRG cells at the mRNA level and CYP activity in response to inducers and their predictivity for the detection of human hepatotoxins. Cell Biol Toxicol 28(2):69-87

Hou TJ, Zhang W, Xia K, Qiao XB, Xu XJ (2004) ADME evaluation in drug discovery. 5. Correlation of Caco-2 permeation with simple molecular properties. J Chem Inf Comput Sci 44(5):1585-1600

IARC (2017) Agents Classified by the IARC Monographs, vol 1-118. International Agency for Research on Cancer, France

Ingelman-Sundberg M (2004) Human drug metabolising cytochrome P450 enzymes: properties and polymorphisms. Naunyn Schmiedebergs Arch Pharmacol 369(1):89-104

Kanebratt KP, Andersson TB (2008) Evaluation of HepaRG cells as an in vitro model for human drug metabolism studies. Drug Metab Dispos 36(7):1444-1452

Kvist AJ, Kanebratt KP, Walentinsson A, Palmgren H, O'Hara M, Bjorkbom A, Andersson LC, Ahlqvist M, Andersson TB (2018) Critical differences in drug metabolic properties of human hepatic cellular models, including primary human hepatocytes, stem cell derived hepatocytes, and hepatoma cell lines. Biochem Pharmacol 155:124-140

Lambert CB, Spire C, Claude N, Guillouzo A (2009) Dose- and timedependent effects of phenobarbital on gene expression profiling in human hepatoma HepaRG cells. Toxicol Appl Pharmacol 234(3):345-360

Lame MW, Morin D, Jones AD, Segall HJ, Wilson DW (1990) Isolation and identification of a pyrrolic glutathione conjugate 
metabolite of the pyrrolizidine alkaloid monocrotaline. Toxicol Lett 51(3):321-329

Li N, Xia QS, Ruan JQ, Fu PP, Lin G (2011) Hepatotoxicity and tumorigenicity induced by metabolic activation of Pyrrolizidine Alkaloids in Herbs. Curr Drug Metab 12(9):823-834

Lin KM, Lau JK, Smith R, Phillips P, Antal E, Poland RE (1988) Comparison of alprazolam plasma levels in normal Asian and Caucasian male volunteers. Psychopharmacology 96(3):365-369

Lin G, Cui YY, Hawes EM (1998) Microsomal formation of a pyrrolic alcohol glutathione conjugate of clivorine. Firm evidence for the formation of a pyrrolic metabolite of an otonecine-type pyrrolizidine alkaloid. Drug Metab Dispos 26(2):181-184

Lubberstedt M, Muller-Vieira U, Mayer M, Biemel KM, Knospel F, Knobeloch D, Nussler AK, Gerlach JC, Zeilinger K (2011) HepaRG human hepatic cell line utility as a surrogate for primary human hepatocytes in drug metabolism assessment in vitro. J Pharmacol Toxicol 63(1):59-68

Medinsky MA, Leavens TL, Csanady GA, Gargas ML, Bond JA (1994) In vivo metabolism of butadiene by mice and rats: a comparison of physiological model predictions and experimental data. Carcinogenesis 15(7): 1329-1340

Miranda CL, Reed RL, Guengerich FP, Buhler DR (1991) Role of cytochrome P450IIIA4 in the metabolism of the pyrrolizidine alkaloid senecionine in human liver. Carcinogenesis 12(3):515-519

Mohabbat O, Younos MS, Merzad AA, Srivastava RN, Sediq GG, Aram GN (1976) An outbreak of hepatic veno-occlusive disease in north-western Afghanistan. Lancet 2(7980):269-271

NHFPC (2007) Reference individuals for use in radiation protectionPart 2: Masses of main organs and tissues. National Health and Family Planning Commission of the People's Republic of China

NHFPC (2014) Reference individuals for use in radiation protectionPart 3: main physiological parameters. vol GBZ/T 200.3. National Health and Family Planning Commission of the People's Republic of China

Ning J, Louisse J, Spenkelink B, Wesseling S, Rietjens IMCM (2017) Study on inter-ethnic human differences in bioactivation and detoxification of estragole using physiologically based kinetic modeling. Arch Toxicol 91(9):3093-3108

NTP (1978) Bioassay of lasiocarpine for possible carcinogenicity. Natl Cancer Inst Carcinog Tech Rep Ser 39:1-66

NTP (2003) Toxicology and carcinogenesis studies of riddelliine (CAS No. 23246-96-0) in F344/N rats and B6C3F1 mice (gavage studies). Natl Toxicol Program Tech Rep Ser(508)

Paini A, Punt A, Viton F, Scholz G, Delatour T, Marin-Kuan M, Schilter B, van Bladeren PJ, Rietjens IMCM (2010) A physiologically based biodynamic (PBBD) model for estragole DNA binding in rat liver based on in vitro kinetic data and estragole DNA adduct formation in primary hepatocytes. Toxicol Appl Pharmacol 245(1):57-66

Punt A, Paini A, Boersma MG, Freidig AP, Delatour T, Scholz G, Schilter B, van Bladeren PJ, Rietjens IMCM (2009) Use of physiologically based biokinetic (PBBK) modeling to study estragole bioactivation and detoxification in humans as compared with male rats. Toxicol Sci 110(2):255-269

Reed RL, Miranda CL, Kedzierski B, Henderson MC, Buhler DR (1992) Microsomal formation of a pyrrolic alcohol glutathione conjugate of the pyrrolizidine alkaloid senecionine. Xenobiotica 22(11):1321-1327

Shi ZR, Yan LN, Li B, Wen TF (2009) Evaluation of standard liver volume formulae for Chinese adults. World J Gastroenterol 15(32):4062-4066

Slob W (2002) PROAST: Software for dose-response modeling and benchmark dose analysis. RIVM

Smith LW, Culvenor CCJ (1981) Plant sources of hepatotoxic pyrrolizidine alkaloids. J Nat Prod 44(2):129-152
Stegelmeier BL, Edgar JA, Colegate SM, Gardner DR, Schoch TK, Coulombe RA, Molyneux RJ (1999) Pyrrolizidine alkaloid plants, metabolism and toxicity. J Nat Toxins 8(1):95-116

Tamta H, Pawar RS, Wamer WG, Grundel E, Krynitsky AJ, Rader JI (2012) Comparison of metabolism-mediated effects of pyrrolizidine alkaloids in a HepG2/C3A cell-S9 co-incubation system and quantification of their glutathione conjugates. Xenobiotica 42(10):1038-1048

Tandon HD, Tandon BN, Mattocks AR (1978) An epidemic of venoocclusive disease of the liver in Afghanistan. Pathologic features. Am J Gastroenterol 70(6):607-613

Tu M, Sun S, Wang K, Peng X, Wang R, Li L, Zeng S, Zhou H, Jiang H (2013) Organic cation transporter 1 mediates the uptake of monocrotaline and plays an important role in its hepatotoxicity. Toxicology 311(3):225-230

van Liempd S, Morrison D, Sysmans L, Nelis P, Mortishire-Smith R (2011) Development and validation of a higher-throughput equilibrium dialysis assay for plasma protein binding. J Lab Autom 16(1):56-67

Wang D, Zou L, Jin Q, Hou J, Ge G, Yang L (2018) Human carboxylesterases: a comprehensive review. Acta Pharmaceutica Sinica $\mathrm{B}$ (In press)

Waters NJ, Jones R, Williams G, Sohal B (2008) Validation of a rapid equilibrium dialysis approach for the measurement of plasma protein binding. J Pharm Sci 97(10):4586-4595

Westlind-Johnsson A, Malmebo S, Johansson A, Otter C, Andersson TB, Johansson I, Edwards RJ, Boobis AR, Ingelman-Sundberg M (2003) Comparative analysis of CYP3A expression in human liver suggests only a minor role for CYP3A5 in drug metabolism. Drug Metab Dispos 31(6):755-761

WHO (1999) International Programme on Chemical Safety (IPCS)? Principles for the assessment of risks to human health from exposure to chemicals. World Health Organization, Geneva

Wiedenfeld H, Edgar J (2011) Toxicity of pyrrolizidine alkaloids to humans and ruminants. Phytochem Rev 10(1):137-151

Wilkinson GR (2005) Drug therapy-Drug metabolism and variability among patients in drug response. New Engl J Med 352(21):2211-2221

Xia Q, Chou MW, Kadlubar FF, Chan PC, Fu PP (2003) Human liver microsomal metabolism and DNA adduct formation of the tumorigenic pyrrolizidine alkaloid, riddelliine. Chem Res Toxicol 16(1):66-73

Xia Q, Zhao Y, Von Tungeln LS, Doerge DR, Lin G, Cai L, Fu PP (2013) Pyrrolizidine alkaloid-derived DNA adducts as a common biological biomarker of pyrrolizidine alkaloid-induced tumorigenicity. Chem Res Toxicol 26(9):1384-1396

Yang JL, He MM, Niu W, Wrighton SA, Li L, Liu Y, Li C (2012) Metabolic capabilities of cytochrome $\mathrm{P} 450$ enzymes in Chinese liver microsomes compared with those in Caucasian liver microsomes. Brit J Clin Pharmaco 73(2):268-284

Yuan D, Lu T, Wei YG, Li B, Yan LN, Zeng Y, Wen TF, Zhao JC (2008) Estimation of standard liver volume for liver transplantation in the Chinese population. Transplant Proc 40(10):3536-3540

Zhao Y, Xia Q, Gamboa da Costa G, Yu H, Cai L, Fu PP (2012) Full structure assignments of pyrrolizidine alkaloid DNA adducts and mechanism of tumor initiation. Chem Res Toxicol 25(9):1985-1996

Publisher's Note Springer Nature remains neutral with regard to jurisdictional claims in published maps and institutional affiliations. 